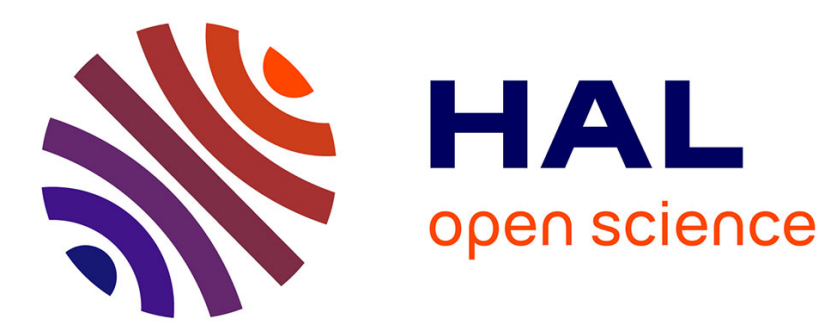

\title{
Generalized Choquet-like aggregation functions for handling bipolar scales
}

Christophe Labreuche, Michel Grabisch

\section{To cite this version:}

Christophe Labreuche, Michel Grabisch. Generalized Choquet-like aggregation functions for handling bipolar scales. European Journal of Operational Research, 2006, 172 (3), pp.931-955. 10.1016/j.ejor.2004.11.008 . halshs-00186907

\section{HAL Id: halshs-00186907 https://shs.hal.science/halshs-00186907}

Submitted on 12 Nov 2007

HAL is a multi-disciplinary open access archive for the deposit and dissemination of scientific research documents, whether they are published or not. The documents may come from teaching and research institutions in France or abroad, or from public or private research centers.
L'archive ouverte pluridisciplinaire HAL, est destinée au dépôt et à la diffusion de documents scientifiques de niveau recherche, publiés ou non, émanant des établissements d'enseignement et de recherche français ou étrangers, des laboratoires publics ou privés. 


\title{
Generalized Choquet-like aggregation functions for handling bipolar scales
}

\author{
Christophe Labreuche ${ }^{\dagger}$ \\ Thales Research \& Technology \\ Domaine de Corbeville \\ 91404 Orsay Cedex, France \\ Michel Grabisch \\ Université Paris I Panthéon-Sorbonne \\ LIP6, 8 rue du Capitaine Scott, \\ 75015 Paris, France
}

\begin{abstract}
We are interested in modeling interaction between criteria in MultiCriteria Decision Making when underlying scales are bipolar. Interacting phenomena involving behavioral bias between attractive and repulsive values are in particular considered here. We show in an example that both the Choquet integral and the Cumulative Prospect Theory (CPT) model fail to represent these interacting phenomena. Axioms that enable the construction of the preferences of the decision maker over each attribute, and the representation of his preferences about aggregation of criteria are introduced and justified. We show there is a unique aggregation operator that fits with these axioms. It is based on the notion of bi-capacity and generalizes both the Choquet integral and the CPT model.
\end{abstract}

Keywords: decision support systems, multiple criteria analysis, Choquet integral, capacities, axiomatic approach.

\footnotetext{
${ }^{\dagger}$ Corresponding author.

E-mail address: christophe.labreuche@thalesgroup.com
} 


\section{Introduction}

Multi-criteria decision making (MCDM) is a branch of decision theory where acts or alternatives are chosen considering several points of view or criteria, assuming that the decision maker (DM) has all the information at his/her disposal concerning the alternatives, i.e. they are fully described by a vector of attributes which is supposed to be known without uncertainty. Two main features of this kind of problem make it difficult to solve. The first one is that attributes describing alternatives are heterogeneous, i.e. they represent different physical (or economical, subjective, ... ) entities like price, size, color, weight, etc. and may be numerical or not. Hence a first difficulty is to make them commensurable in some sense. The second feature is that points of view or criteria are more or less important to make decision, and most often they are conflicting or interacting in some way, so that it is not obvious how to combine them for making a final overall opinion.

Many approaches to MCDM have been proposed so far, among which outranking approaches and multi-attribute utility theory (MAUT) approaches are most representative [30]. Outranking approaches easily solve the commensurateness problem by making pairwise comparisons, however the final decision stage is hindered by the problem of combining preference relations, and thus comes up against Arrow's theorem. MAUT approaches [20] rely on the construction of utility functions, which can be fairly difficult because of commensurateness problems, but then easily reach a final decision by combining utilities or scores of all criteria. Our approach is also based on MAUT philosophy.

In most approaches criteria are combined by taking a weighted average, and this implicitly prevents the modeling of interactions between those criteria and conjunctive/disjunctive behavior. The use of the Choquet integral w.r.t. a capacity as aggregation function, permits the representation of some interaction among criteria [12]. For a complete construction of a MCDM method based on the Choquet integral we refer to [23].

A further relevant aspect relates to the type of scale underlying scores or utilities. Studies in psychology (see e.g. Osgood et al. [28]) have shown that often the scales used to represent scores or utilities should be considered as bipolar, since decision making is often guided by affect. According to Slovic [35], affect is 'the specific quality of 'goodness' and 'badness', as felt consciously or not by the decision maker, and demarcating a positive or negative quality of stimulus". Then it is natural to use a scale going from negative (bad) to positive (good) values, including a central neutral value, to encode the bipolarity of the affect. Such a scale is called a bipolar scale, typical examples where the neutral value is taken as 0 being $[-1,1]$ or $\mathbb{R}$. 
Therefore, it is important to derive models taking into account this dichotomy. The Choquet integral, which is invariant to a shift of the scale, is insensitive to the neutral level, and thus cannot serve for this purpose. However, one can add some sensitivity by a simple symmetrization of the Choquet integral around 0 , or more generally one can compute the positive scores and the negative ones separately by two Choquet integrals w.r.t different capacities, thus enabling to model a different behavior of the decision maker when faced with positive or negative scores. This is in fact the Cumulative Prospect Theory (CPT) model, proposed by Tversky and Kahnemann [37], in the field of decision under risk.

Although very general, the CPT model may fail to represent some behavior, especially when alternatives have at the same time "good" scores (above the neutral level) on some criteria and "bad" scores on other ones. Then if some interaction occurs between the criteria with good scores (satisfied criteria) and the ones with bad scores (unsatisfied criteria), the CPT model is not able to represent the preference of the DM (such an example will be presented in Section 3). The reason is that in this model, positive and negative scores are aggregated separately.

In [16], the notion of bi-capacity is proposed to resolve this problem. Roughly speaking, a bi-capacity encodes the score of all possible combinations of satisfied and unsatisfied criteria, so that it is able to represent complex interaction phenomena. Then, the Choquet integral defined with respect to a bi-capacity should enable the computation of the overall score of any alternative, and encompass the CPT model.

The aim of this paper is precisely to introduce this generalized Choquet integral, to prove that it effectively solves situations where CPT fails, and to provide an axiomatization and construction of a MCDM model using similar techniques as presented in [23] for the Choquet integral.

The paper is organized as follows. Section 2 gives the necessary background on capacities, bi-capacities, the Choquet integral and the CPT model. Section 3 describes a simple example of interaction between criteria, which can be modeled by bi-capacities but not by the CPT model. The Choquet integral w.r.t. a bi-capacity is constructed in Section 4 from some intracriterion and inter-criteria information asked to the DM. It is shown that this new operator generalizes the CPT model. In section 5, we discuss on the practicality of our approach. The determination of the reference levels necessary to ensure commensurateness among criteria is discussed in Section 5.1. Section 5.2 describes the way utility functions $u_{i}$ can be constructed in practice. Lastly, the determination of bi-capacities is discussed in Section 5.3 . 


\section{Preliminaries}

\subsection{General framework for MCDM}

We denote by $X_{1}, \ldots, X_{n}$ the sets of possible values taken by representative attributes faithfully describing alternatives of interest, and by $N:=$ $\{1, \ldots, n\}$ the index set of attributes. $X:=X_{1} \times \cdots \times X_{n}$ is the set of potential alternatives, and we denote by $\succeq$ a binary relation on $X$ expressing the preference of the DM. The aim is to get a representation of $\succeq$ on $X$ on the basis of some information provided by the DM only on a subset $\mathcal{A}$ of $X$. This representation takes the form of a function $u: X \longrightarrow \mathbb{R}$ such that for any $x, y \in \mathcal{A}, x \succeq y$ if and only if $u(x) \geq u(y)$. The most common form for $u$ is the decomposable form:

$$
u(x)=F\left(u_{1}\left(x_{1}\right), \ldots, u_{n}\left(x_{n}\right)\right) \quad \forall x \in X,
$$

and general conditions on $\succeq$ and $X$ are known for such a model to exist [22]. The functions $u_{i}: X_{i} \longrightarrow \mathbb{R}$ are called the utility functions, while $F: \mathbb{R}^{n} \longrightarrow \mathbb{R}$ is called the aggregation function. Each utility function $u_{i}$ induces a complete preorder $\succeq_{i}$ on $X_{i}$. Roughly speaking, utility functions assign scores to each possible values of the attribute, and they have to be commensurate, in the sense that a given score on two different attributes means the same satisfaction for the DM. We call criterion an attribute with its utility function (and by abuse, the attribute itself).

The construction of the utility functions and the determination of the aggregation function are often carried out in two separate steps. The utility functions are generally set up first, that is without the knowledge of the aggregation function $F$. However, the utility functions have no intrinsic meaning to the DM and shall be determined from questions regarding only the overall preference relation $\succeq$. It is not assumed that the DM can isolate attributes and give information directly on $u_{i}$. This point is generally not considered in the literature. The main reason is probably that due to the use of a weighted sum as an aggregation function, the independence assumption (preferential or cardinal independence) makes it possible in some sense

to separate each attribute, and thus directly construct the utility functions. This becomes far more complicated when this assumption is removed. Besides, these approaches are not relevant from a theoretical standpoint. To our knowledge, the only approach that addresses this problem with the use of a weighted sum is the so-called Macbeth approach designed by C. Bana e Costa and J.C. Vansnick $[1,2,3,4]$. A generalization of this approach to the Choquet integral has been proposed in [23]. The Macbeth approach is also considered in this paper. 
Considering two alternatives $x, y \in X$ and $A \subset N$, we use the notation $\left(x_{A}, y_{-A}\right)$ to denote the compound alternative $z \in X$ such that $z_{i}=x_{i}$ if $i \in A$ and $y_{i}$ otherwise. Similarly for two real vectors of scores $w, t \in \mathbb{R}^{n}$, we denote by $\left(w_{A}, t_{-A}\right)$ the compound vector $w^{\prime} \in \mathbb{R}^{n}$ such that $w_{i}^{\prime}=w_{i}$ for $i \in A$ and $t_{i}$ for $i \notin A$.

\subsection{Bipolar and unipolar scales}

It may exist in $X_{i}$ a particular element or level $\mathbf{0}_{i}$, called neutral level, such that if $x_{i} \succ_{i} \mathbf{0}_{i}$, then $x_{i}$ is considered as "good", while if $x_{i} \prec_{i} \mathbf{0}_{i}$, then $x_{i}$ is considered as "bad" for the DM.

Such a neutral level exists whenever relation $\succeq_{i}$ corresponds to two opposite notions of common language. For example, this is the case when $\succeq_{i}$ means "more attractive than", "better than", etc., whose pairs of opposite notions are respectively "attractiveness/repulsiveness", and "good/bad". By contrast, relations such as "more allowed than", "belongs more to category $C$ than" do not clearly exhibit a neutral level.

A scale is said to be bipolar if $X_{i}$ contains such a neutral level. A unipolar scale has no neutral level, but has a least level, i.e. an element or level denoted also by $\mathbf{0}_{i}$ in $X_{i}$ such that $x_{i} \succeq_{i} \mathbf{0}_{i}$ for all $x_{i} \in X_{i}$.

The bipolar nature of a scale is often quite debatable, especially in MCDM. In MCDM, the criteria have the meaning of some kind of satisfaction. The $[0,1]$ unipolar representation of criteria classically used in MCDM has the interpretation of a satisfaction degree. The two bounds of this scale depict "not satisfied at all" and "perfectly satisfied" values respectively. However, values around 0 are clearly "bad", whereas values around 1 are clearly "good". The $[0,1]$ unipolar scale can thus be seen as a bipolar scale, with for instance the neutral value $\frac{1}{2}$. In Fuzzy Sets Theory, uninorms (mixing a $t$-norm on the ill-satisfied situations and a $t$-conorm on the well-satisfied situations [21]) are examples of bipolar operators applied to the fuzzy interval $[0,1]$. The crossover point in uninorms corresponds to the neutral level in bipolar scales. To sum-up, scales used in MCDM can be viewed as either unipolar or bipolar due to the particular meaning alloted to these scales. So, the choice of the type of scale depends more on the choice of the reference points used on the attributes than the true meaning of the attributes. We advocate the use of bipolar scales if the DM behaves differently when faced to well-satisfied compared to ill-satisfied values. Due to commensurateness between criteria, one should use the same scale (and thus the same reference levels) on all criteria. This suggests that if one attribute requires a bipolar scale, all attributes need to be treated as bipolar. We assume henceforth that all scales are bipolar. We make the assumption that the neutral elements $\mathbf{0}_{i}$ exist on all scales. 


\subsection{Aggregation on unipolar scales}

In Cooperative Game Theory, a game on $N$ is defined as a set function that vanishes on the empty set. Let $\mathcal{G}^{1}(N)$ be the set of all games (also called improper games) [29]:

$$
\mathcal{G}^{1}(N)=\left\{\nu: 2^{N} \rightarrow \mathbb{R}, \quad \nu(\emptyset)=0\right\}
$$

An interesting subclass of games is the ones that are non-decreasing and normalized :

$$
\mathcal{C}^{1}(N)=\left\{\nu \in \mathcal{G}^{1}(N), \quad \forall A \subset B \subset N, \nu(A) \leq \nu(B) \text { and } \nu(N)=1\right\}
$$

Elements of $\mathcal{C}^{1}(N)$ are called capacities [7] or equivalently fuzzy measures [13]. In MCDM, $\nu(A)$ is interpreted as the overall assessment of the "binary" vector of scores on criteria $\left(1_{A}, 0_{-A}\right)$. Since the same scale is used for all criteria and for the output assessment, one has $\nu(\emptyset)=0$ and $\nu(N)=1$. Moreover, since this commensurate scale is a satisfaction scale (hence has the following meaning: "the larger the better"), $\nu$ is non-decreasing. Set functions considered in MCDM are thus capacities. However, as we will see later, bi-capacities are related to games that are neither non-decreasing nor normalized.

The Choquet integral of $w=\left(w_{1}, \ldots, w_{n}\right) \in \mathbb{R}^{n}$ defined w.r.t. a game $\nu \in \mathcal{G}^{1}(N)$ has the following expression [7] :

$$
C_{\nu}\left(w_{1}, \ldots, w_{n}\right)=\sum_{i=1}^{n}\left(w_{\tau(i)}-w_{\tau(i-1)}\right) \nu(\{\tau(i), \cdots, \tau(n)\})
$$

where $w_{\tau(0)}:=0$ and $w_{\tau(1)} \leq w_{\tau(2)} \leq \cdots \leq w_{\tau(n)}$.

We say that $w, t \in \mathbb{R}^{n}$ are comonotone if $w_{i}<w_{j} \Rightarrow t_{i} \leq t_{j}$ for any $i, j \in$ $N$. In other words, $w, t$ are comonotone if they belong to $\Gamma_{\tau}:=\left\{w \in \mathbb{R}^{n} \mid\right.$ $\left.w_{\tau(1)} \leq w_{\tau(2)} \leq \cdots \leq w_{\tau(n)}\right\}$ for the same permutation $\tau$. Thus, it is clear from (2) that for comonotone acts $w, t$ we have $C_{\mu}(w+t)=C_{\mu}(w)+C_{\mu}(t)$. This property, called comonotonic additivity, is characteristic of the Choquet integral, as shown by Schmeidler [31]. Hence the Choquet integral acts like a weighted sum in each $\Gamma_{\tau}$ (for a fixed $\tau$ ).

Let us now give an axiomatic justification of the Choquet integral on interval scales (i.e. scales defined up to a shift and dilation) in the context of MCDM. The main result comes from Marichal [25]. It characterizes the Choquet integral as an aggregation function in $\mathbb{R}^{n}$. This result has been 
improved by using a weaker axiom [23]. We present this characterization. Since it has been done in the MCDM context, it concerns only capacities.

Consider a function $F_{\nu}: \mathbb{R}^{n} \rightarrow \mathbb{R}$ (indexed by a capacity $\nu \in \mathcal{C}^{1}(N)$ ) dedicated to the aggregation of criteria on interval scales. So, $F_{\nu}$ shall satisfy some reasonable conditions.

Linearity w.r.t. Capacities (LC): For any $\nu, \nu_{1}, \ldots, \nu_{p} \in$ $\mathcal{C}^{1}(N)$ such that $\nu=\sum_{i=1}^{p} \alpha_{i} \nu_{i}$, with $\alpha_{1}, \ldots, \alpha_{p} \in \mathbb{R}$, it holds for all $w \in \mathbb{R}^{n}$

$$
F_{\nu}(w)=\sum_{i=1}^{p} \alpha_{i} F_{\nu_{i}}(w)
$$

(LC) is equivalent to the linearity axiom given in [25]. This latter states that there exists $2^{n}$ functions $F_{A}: \mathbb{R}^{n} \rightarrow \mathbb{R}$ (for all $A \subset N$ ) such that $F_{\nu}=\sum_{A \subset N} \nu(A) F_{A}$. Marichal motivates his axiom saying that, in order to keep the aggregation model as simple as possible, $F_{\nu}(w)$ (even though not linear w.r.t. $w)$ is assumed to be linear w.r.t. $\nu$.

(LC) can be explained in the following way. It is usual in MCDM to write a capacity $\nu$ as a linear combination of elementary capacities $\nu_{i}$. Such elementary capacities correspond to typical decision strategies. For instance, a two-additive capacity [13] can be viewed as a convex combination of the following three types of typical decision strategies: extreme intolerance and tolerance between two criteria (leading to the minimum and the maximum of the two criteria scores respectively), and dictatorship w.r.t. a criterion. A capacity can also be written in terms of the unanimity games [13], using the Möbius transform. Other decompositions are also possible [13]. Since the elementary decision strategies are very intuitive for a DM, one would like to define the aggregation function $F_{\nu_{i}}$ for these strategies and deduce the overall function $F_{\nu}$ by applying the same decomposition to the $F_{\nu_{i}}$ 's. This yields (LC). In the example of the decomposition w.r.t. the unanimity games, the coefficients may be negative, which explains why one has $\alpha_{1}, \ldots, \alpha_{p} \in \mathbb{R}$ in (LC).

Monotonicity w.r.t. Capacities (MC): For any $\nu \in \mathcal{C}^{1}(N)$ and $\forall w, t \in \mathbb{R}^{n}$,

$$
w_{i} \geq t_{i}, \forall i \in N \Rightarrow F_{\nu}(w) \geq F_{\nu}(t)
$$


Increasingness of the aggregation function is a natural requirement [10]. In MCDM, all criteria are given on the same commensurate scale, and this latter depicts the satisfaction degree of the DM. Hence, if an alternative $w$ has better partial scores than another one $t$, then $w$ is preferred to $t$ over each criterion. Hence, $w$ is at least as good as $t$ for the DM, so that $F_{\nu}(w) \geq F_{\nu}(t)$. This is Pareto-optimality. We have already seen that non-decreasingness of a game is also justified by the same argument. Increasingness of $F_{\nu}$ can only be obtained when $\nu$ is non-decreasing.

Properly Weighted w.r.t. Capacities (PWC): For any $\nu \in$ $\mathcal{C}^{1}(N), F_{\nu}\left(1_{A}, 0_{-A}\right)=\nu(A), \forall A \subset N$.

For a weighted sum $F_{\nu}(w)=\sum_{i=1}^{n} \nu(\{i\}) w_{i}$, one clearly has that $F_{\nu}\left(1_{\{i\}}, 0_{-\{i\}}\right)=$ $\nu(\{i\})$ and more generally $F_{\nu}\left(1_{A}, 0_{-A}\right)=\sum_{i \in A} \nu(\{i\})=\nu(A)$, indicating that the weight of a coalition $A$ of criteria is defined as the overall evaluation of the binary vector $\left(1_{A}, 0_{-A}\right)$. The assignment of importance of coalitions of criteria is tightly linked to the evaluation function. We obtain (PWC) naturally.

\section{Stable under Positive Linear transformations for Capac-} ities and binary acts (SPLC): For any $\nu \in \mathcal{C}^{1}(N)$, for all $A \subset N, \alpha>0$, and $\beta \in \mathbb{R}$,

$$
F_{\nu}\left((\alpha+\beta)_{A}, \beta_{-A}\right)=\alpha F_{\nu}\left(1_{A}, 0_{-A}\right)+\beta
$$

The interval scale associated to a criterion $i$ is given up to a positive linear transformation $\phi_{i}\left(w_{i}\right)=r_{i} w_{i}+s_{i}$, with $r_{i}>0$ and $s_{i} \in \mathbb{R}[22]$. From a measurement standpoint, the aggregation function shall be stable under positive linear transformations : $F_{\nu}\left(r_{1} w_{1}+s_{1}, \ldots, r_{n} w_{n}+s_{n}\right)=r F_{\nu}\left(w_{1}, \ldots, w_{n}\right)+s$, where $\phi(f)=r f+s$ is the transformation applied to the overall evaluation. Since all scales are commensurate, one may only apply the same transformation to all scales [25], leading to :

$$
F_{\nu}\left(r w_{1}+s, \ldots, r w_{n}+s\right)=r F_{\nu}\left(w_{1}, \ldots, w_{n}\right)+s
$$

The original axiom from Marichal indicates that (3) holds for all $w=\left(w_{1}\right.$, $\left.\ldots, w_{n}\right) \in \mathbb{R}^{n}, r>0$ and $s \in \mathbb{R}$. Axiom (SPLC) is weaker in the sense that (3) is required only on the binary acts.

We have the following result [23]. 
Theorem $1\left\{F_{\nu}\right\}_{\nu \in \mathcal{C}^{1}(N)}$ satisfies (LC), (MC), (PWC) and (SPLC) if and only if for any $\nu \in \mathcal{C}^{1}(N)$, we have $F_{\nu}(w)=C_{\nu}(w)$ for all $w \in \mathbb{R}^{n}$.

We now give a characterization of the Choquet integral for games instead of being restricted to capacities. Moreover we consider only positive scores. This result will be useful for bi-capacities. We introduce the following axioms. These are straightforward modifications of axioms (LC), (MC) and (SPLC). We extend only the linearity axiom on games.

Linearity w.r.t. Games $\left(\mathbf{L G}^{+}\right)$: For any $\nu, \nu_{1}, \ldots, \nu_{p} \in \mathcal{G}^{1}(N)$ such that $\nu=\sum_{i=1}^{p} \alpha_{i} \nu_{i}$, with $\alpha_{1}, \ldots, \alpha_{p} \in \mathbb{R}$, it holds for all $w \in \mathbb{R}_{+}^{n}$

$$
F_{\nu}(w)=\sum_{i=1}^{p} \alpha_{i} F_{\nu_{i}}(w)
$$

Monotonicity w.r.t. Capacities for positive acts $\left(\mathrm{MC}^{+}\right)$: For any $\nu \in \mathcal{C}^{1}(N)$ and $\forall w, t \in \mathbb{R}_{+}^{n}$,

$$
w_{i} \geq t_{i}, \forall i \in N \Rightarrow F_{\nu}(w) \geq F_{\nu}(t) .
$$

Stable under Positive Linear transformations with positive shifts for Capacities and binary acts $\left(\mathrm{SPLC}^{+}\right)$: For any $\nu: \mathcal{C}^{1}(N)$, for all $A \subset N, \alpha>0$, and $\beta \in \mathbb{R}_{+}$,

$$
F_{\nu}\left((\alpha+\beta)_{A}, \beta_{-A}\right)=\alpha F_{\nu}\left(1_{A}, 0_{-A}\right)+\beta
$$

Theorem $2\left\{F_{\nu}\right\}_{\nu \in \mathcal{G}^{1}(N)}$ satisfies $\left(\mathbf{L G}^{+}\right),\left(\mathbf{M C}^{+}\right),(\mathbf{P W C})$ and $\left(\mathbf{S P L C}^{+}\right)$ if and only if for any $\nu \in \mathcal{G}^{1}(N), F_{\nu}(w)=C_{\nu}(w)$ for all $w \in \mathbb{R}_{+}^{n}$.

One observes that a characterization of the Choquet integral in $\mathbb{R}^{n}$ for games results by replacing (LC) in Theorem 2 with linearity w.r.t. a game. The proof is based on the following result.

Lemma 1 If $F: \mathbb{R}_{+}^{n} \rightarrow \mathbb{R}$ satisfies

(i) $F$ is non-decreasing in $\mathbb{R}_{+}^{n}$

(ii) $F\left((\alpha+\beta)_{A}, \beta_{-A}\right)=\alpha F\left(1_{A}, 0_{-A}\right)+\beta$ for any $A \subset N, \alpha>0$ and $\beta \in \mathbb{R}_{+}$ 
(iii) $F\left(1_{A}, 0_{-A}\right) \in\{0,1\}$ for all $A \subset N$

(iv) $F\left(0_{N}\right)=0$ and $F\left(1_{N}\right)=1$

then $F(w)=C_{v}(w)$ for all $w \in \mathbb{R}_{+}^{n}$, where $v$ is a capacity given by $v(A)=$ $F\left(1_{A}, 0_{-A}\right)$ for all $A \subset N$.

This lemma is a slight modification of the following one.

Lemma 2 (Lemma 2 in [23]) If $F: \mathbb{R}^{n} \rightarrow \mathbb{R}$ satisfies

(i) $F$ is non-decreasing in $\mathbb{R}^{n}$

(ii) $F\left((\alpha+\beta)_{A}, \beta_{-A}\right)=\alpha F\left(1_{A}, 0_{-A}\right)+\beta$ for any $A \subset N, \alpha>0$ and $\beta \in \mathbb{R}$

(iii) $F\left(1_{A}, 0_{-A}\right) \in\{0,1\}$ for all $A \subset N$

(iv) $F\left(0_{N}\right)=0$ and $F\left(1_{N}\right)=1$

then $F(w)=C_{v}(w)$ for all $w \in \mathbb{R}^{n}$, where $v$ is a capacity given by $v(A)=$ $F\left(1_{A}, 0_{-A}\right)$ for all $A \subset N$.

Proof of Lemma 1: The only difference between Lemmas 1 and 2 except the restriction of $\mathbb{R}_{+}^{n}$ is the sign of $\beta$ in (ii). When we restrict ourself to acts $w$ in $\mathbb{R}_{+}^{n}$, condition (ii) in Lemma 2 is used only with positive values of $\beta$. Henceforth, condition (ii) in Lemma 2 can be replaced by condition (ii) in Lemma 1.

Proof of Theorem 2: The proof is similar to that of Theorem 1 in [23] which relies on Lemma 2. Lemma 2 is replaced here by Lemma 1 . Moreover, we use the decomposition of a game in terms of the unanimity games. Function $F_{\nu}$ where $\nu$ is a unanimity game satisfies all conditions of Lemma 1.

Let us conclude this section by describing the Macbeth approach on unipolar scales [23]. The interval scale on criterion $i$ is given up to a positive linear transformation $\phi_{i}\left(w_{i}\right)=r_{i} w_{i}+s_{i}$, (with $r_{i}>0$ and $s_{i} \in \mathbb{R}$ ) that normalizes the scale. The non-normalized interval scales are constructed independently (intra-criterion construction). In order to fix the two degrees of freedom $r_{i}$ and $s_{i}$ on each scale, two reference levels that have the same meaning throughout the criteria are introduced. It is assumed that there exists an element denoted by $\mathbf{0}_{i}$ which is considered as completely unsatisfactory for the DM, and an element denoted by $\mathbf{1}_{i}$ that is considered as good and completely satisfactory, even if more attractive elements could exist on 
this point of view [23]. The scales are normalized by using the appropriate transformations $\phi_{i}$ so as to enforce commensurateness between the levels, that is $u_{1}\left(\mathbf{0}_{1}\right)=\cdots=u_{n}\left(\mathbf{0}_{n}\right)$ and $u_{1}\left(\mathbf{1}_{1}\right)=\cdots=u_{n}\left(\mathbf{1}_{n}\right)$. This implies the overall commensurateness between the criteria.

We will use different reference levels for bipolar scales but the approach will be similar.

\subsection{Aggregation on bipolar scales}

The previous section has shown an important property of the Choquet integral, namely the (PWC) property, saying that for any binary vector $\left(1_{A}, 0_{-A}\right)$, it holds that $C_{\nu}\left(1_{A}, 0_{-A}\right)=\nu(A)$. This shows that binary vectors are prototype vectors for unipolar scales, since the model is entirely determined from the overall score assigned to these vectors.

Turning to bipolar scales, the question arises on how to score alternatives having a "negative" (i.e. below the neutral level) score on some criteria, in particular ternary vectors $\left(1_{A},-1_{B}, 0_{-(A \cup B)}\right)$, whose components take values 1 on $A,-1$ on $B$, and 0 elsewhere. Several solutions are possible.

The first one is to keep the formula of Choquet integral (2) as it is. Since the Choquet integral commutes with positive affine transformations (i.e. axiom (SPLC) extended to any vector of scores), no particular value of the scale can play a particular role, and so the bipolarity of the scale will be ignored.

The second solution is to aggregate separately positive and negative scores. Assuming a symmetry in the model between the positive and negative parts of the scale, we are led to the so-called symmetric Choquet integral [8] or Šipoš integral [36]:

$$
\check{S}_{\nu}(w):=C_{\nu}\left(w^{+}\right)-C_{\nu}\left(w^{-}\right),
$$

where $w^{+}$and $w^{-}$are respectively the positive and negative parts of $w$, i.e. $\left(w^{+}\right)_{i}:=\max \left(w_{i}, 0\right),\left(w^{-}\right)_{i}:=\max \left(-w_{i}, 0\right)$. The properties of the Šipoš integral in the MCDM framework are given in $[14,15]$.

The symmetry of the above model may be not suitable in certain situations of decision making (e.g. decision under risk or uncertainty, where the behavior of individuals is fairly different for gains and losses). Tversky and Kahnemann have proposed such a generalization [37], known as the Cumulative Prospect Theory (CPT) model:

$$
C P T(w):=C_{\nu_{1}}\left(w^{+}\right)-C_{\nu_{2}}\left(w^{-}\right)
$$


where $\nu_{1}$ and $\nu_{2}$ are two capacities. For properties and characterizations of the CPT model, we refer the reader to the references $[37,38,39,6]$.

As a simple example with three criteria will show, despite its generality, the CPT model may fail to represent preferences of the DM, when complex interaction between attributes occur. To give the intuition for this, we look at the way ternary vectors are scored by the CPT model. Applying (4) to a ternary vector $\left(1_{A},-1_{B}, 0_{-(A \cup B)}\right)$, we get:

$$
C P T\left(1_{A},-1_{B}, 0_{-(A \cup B)}\right)=C_{\nu_{1}}\left(1_{A}, 0_{A^{c}}\right)-C_{\nu_{2}}\left(1_{B}, 0_{B^{c}}\right)=\nu_{1}(A)-\nu_{2}(B) .
$$

Hence the way a ternary vector is scored is completely determined by the way positive and negative binary vectors are scored, but one may wish to have more freedom: some interaction phenomena could occur between the positive and the negative parts, which may violate the above formula. By analogy with unipolar scales, we may say that ternary vectors are the prototype vectors of the bipolar case, and so the model should be based on the overall score assigned to them. This amounts to define a function $\mu$ which assigns to each pair $(A, B)$ of disjoint subsets $A, B \subset N$ a number in $[-1,1]$, which is the score assigned to the ternary vector $\left(1_{A},-1_{B}, 0_{-(A \cup B)}\right)$. In $\mu(A, B)$, the attributes in $A$ refer to measured satisfaction and the attributes in $B$ to measured dissatisfaction through the individual utilities. Natural conditions are $\mu(N, \emptyset)=1, \mu(\emptyset, N)=-1$ and $\mu(\emptyset, \emptyset)=0$, since there is unanimity on all criteria. Now due to dominance conditions, it is also natural to impose that if $A \subseteq B$, then $\mu\left(A, A^{\prime}\right) \leq \mu\left(B, A^{\prime}\right)$ (more criteria have a good score), and $\mu\left(A^{\prime}, A\right) \geq \mu\left(A^{\prime}, B\right)$ (more criteria have a bad score). A function $\mu$ satisfying all these conditions is called a bi-capacity. Let us state this more formally.

Let

$$
\mathcal{Q}(N)=\{(A, B) \in \mathcal{P}(N) \times \mathcal{P}(N) \mid A \cap B=\emptyset\} .
$$

The generalization of games leads to the notion of bi-game :

$$
\mathcal{G}^{2}(N)=\{\mu: \mathcal{Q}(N) \rightarrow \mathbb{R}, \mu(\emptyset, \emptyset)=0\}
$$

A bi-capacity [16] is a normalized and non-decreasing bi-game

$$
\begin{aligned}
\mathcal{C}^{2}(N) & =\left\{\mu \in \mathcal{G}^{2}(N), A \subset A^{\prime} \Rightarrow \mu(A, B) \leq \mu\left(A^{\prime}, B\right),\right. \\
B & \left.\subset B^{\prime} \Rightarrow \mu(A, B) \geq \mu\left(A, B^{\prime}\right), \mu(N, \emptyset)=1 \text { and } \mu(\emptyset, N)=-1\right\} .
\end{aligned}
$$

As said earlier, $\mu(A, B)$ corresponds to the overall assessment of the ternary vector $\left(1_{A},-1_{B}, 0_{-(A \cup B)}\right)$. There exists thus an isomorphism $\Phi: \mathcal{Q}(N) \rightarrow$ $\{-1,0,1\}^{N}$, defined by

$$
\Phi(A, B)=\left(1_{A},-1_{B}, 0_{-(A \cup B)}\right) .
$$


For $A \subset N$, let $\Sigma_{A}:=\left\{w \in \mathbb{R}^{n}, w_{A} \geq 0, w_{-A}<0\right\}$ and $\Sigma_{A}:=\Sigma_{A} \cap\{-1,0,1\}^{N}$. The Choquet integral w.r.t. a bi-capacity $\mu$ proposed in [16] is now given. For any $A \subset N, w \in \Sigma_{A}$,

$$
B C_{\mu}(w):=C_{\nu_{\mu, A}}\left(w_{A},-w_{-A}\right)
$$

where $\nu_{\mu, A}(C):=\mu(C \cap A, C \backslash A)$.

The axiomatic foundation of the expression of $B C_{\mu}$ will be given in Section 4. The asymmetric Choquet integral, the Šipoš integral and the CPT model are particular cases of the Choquet integral w.r.t. a bi-capacity. This will be shown in Theorems 6, 5 and 4 respectively.

We end this section by some bibliographical remarks. In cooperative game theory, equivalent concepts have been proposed. The first attempt seems to be due to D. Felsenthal and M. Machover, who proposed ternary voting games as a generalization of binary voting games [9]. Ternary voting games are functions from $\mathcal{Q}(N)$ to $\{-1,1\}$ satisfying all conditions of bi-capacities except $\mu(\emptyset, \emptyset)=0$. Binary voting games model the result of a vote when some voters are in favor of the bill and the other voters are against [33]. The main limitation of such games is that they cannot represent decision rules in which abstention is an alternative option to the usual yes and no opinions. This leaded D. Felsenthal and M. Machover to introduce ternary voting games [9]. These voting games can be represented by a function with two arguments, one for the yes voters and the other one for the no voters. This concept was limited by the fact that the output value belongs only to the range $\{-1,1\}$. This has been generalized by J.M. Bilbao et al in [5], yielding the definition of bi-cooperative games. Bi-cooperative games satisfy all conditions of bi-capacities.

The generalization of capacities in MCDM to bipolar scales has been carried out independently in [16] and [19]. The concept of bipolar capacity is defined in [19] as a couple of capacities, one for the positive part and one for the negative part. Bipolar capacities are strictly equivalent to bi-capacities when the Choquet integral is used to interpolate these weights [18].

\section{A motivating example}

\subsection{Description of the example}

The director of a University decides on students who are applying for graduate studies in economics where some prerequisites from school are required. Students are indeed evaluated according to mathematics (M), statistics (S) 
and language skills (L). These three criteria serve as a basis for a preselection of the candidates. The best candidates then have an interview with a jury composed of members of the University to assess their motivation in studying economics.

The preselection relies upon scoring the candidates on the three attributes. These scores are computed from the marks they obtained during their last academic year. The easiest way to preselect the candidates would be to require that the marks are above some thresholds on the three attributes. However, one should not use such basic rule since, for instance, a student that has a weakness in one course but is very good in the other ones deserves being preselected. The values we referred to as thresholds are in fact aspiration levels. Scores above these levels are thus considered as 'rather good', and scores below these levels are considered as 'rather bad'.

In general, the applicants have a strong scientific background so that mathematics and statistics are of more importance to the director. However, good students in the sciences with weak language skills should not be attracted. Besides, mathematics and statistics are to some degree substituable, since, usually, students good at mathematics are also good at statistics. As a consequence, for students good in mathematics, the director prefers a student good at languages to one good at statistics. In other words, when the mark with respect to mathematics is good, the director thinks that languages are more important than statistics. This leads to the following rule

(R1): For a student good at mathematics (M), L is more important than $\mathrm{S}$.

Consider now a student that has a weakness in mathematics. In this case, since the applicants are supposed to have strong scientific skills, a student good in statistics is now preferred to one good in languages. More precisely, we have the following statement

(R2): For a student bad in mathematics (M), S is more important than L.

From rules (R1) and (R2), the weight attached to attributes S and L is conditional on attribute $M$ being in one of the ranges good or bad. The aspiration levels (neither good nor bad) have a central role in the attributes. This suggests to use a bipolar scale with these levels as neutral values. All the marks with respect to the courses are given on the same bipolar scale from -10 to 10 , with neutral value 0 .

Consider the following student $A$ 


\begin{tabular}{|c|c|c|c|}
\hline & mathematics & statistics & languages \\
\hline student $A$ & 4 & 6 & -3 \\
\hline
\end{tabular}

According to rule (R1), student $A$ is highly penalized by his performance in languages. As a consequence, the director would prefer a student (with the same mark in mathematics) who is a little bit better in languages even if the student would be worse in statistics. This means that the director prefers the following student to $A$

\begin{tabular}{|c|c|c|c|}
\hline & mathematics & statistics & languages \\
\hline student $B$ & 4 & 5 & -2 \\
\hline
\end{tabular}

We have thus

$$
A \prec B
$$

Consider the following two students

\begin{tabular}{|c|c|c|c|}
\hline & mathematics & statistics & languages \\
\hline student $C$ & -1 & 6 & -3 \\
\hline student $D$ & -1 & 5 & -2 \\
\hline
\end{tabular}

Following rule (R2), $C$ is preferred to $D$ even though $C$ is very bad in languages.

$$
C \succ D
$$

There is an inversion of preferences between (6) and (7) in the sense that the relative importance of languages compared to statistics depends on the satisfaction level in mathematics. This behavior is a typical example of interaction between criteria.

\subsection{Representation attempt of the example}

For our first attempt to model (6) and (7), let us try with the Choquet integral. We have $C_{\mu}(A)=-3+7 \mu(\{\mathrm{M}, \mathrm{S}\})+2 \mu(\{\mathrm{S}\})$ and $C_{\mu}(B)=-2+$ $6 \mu(\{\mathrm{M}, \mathrm{S}\})+\mu(\{\mathrm{S}\})$. This shows that (6) is equivalent to

$$
\mu(\{\mathrm{M}, \mathrm{S}\})+\mu(\{\mathrm{S}\})<1 .
$$

Similarly, relation (7) is equivalent to

$$
\mu(\{\mathrm{M}, \mathrm{S}\})+\mu(\{\mathrm{S}\})>1,
$$


which contradicts previous inequality. Hence, the Choquet integral cannot model both (6) and (7).

It is no surprise that the Choquet integral cannot model both (R1) and (R2). This is due to the fact that the Choquet integral satisfies additivity to comonotone acts. This property shows that the Choquet integral is able to model rules of the following type :

(R1'): If $\mathrm{M}$ is the best satisfied criteria, $\mathrm{L}$ is more important than S.

(R2'): If $\mathrm{M}$ is the worst satisfied criteria, $\mathrm{S}$ is more important than L.

On the other hand, rules (R1) and (R2) make a reference to absolute values (good/bad in Mathematics). The Choquet integral does not allow to model this type of property. The Choquet integral fails to represent the expertise that makes an explicit reference to an absolute value.

In our example, the marks of the four applicants are ranked in the same way: Languages is the worst score, Mathematics is the second best score, and Statistics is the best score. Those four students are comonotone and thus are represented by the same weighted sum with exactly the same coefficients. Since the weighted sum cannot model interaction, the Choquet integral clearly cannot model (6) and (7).

Bipolar aggregation functions might be more appropriate than the asymmetric Choquet integral. Let us first try the symmetric Choquet integral. We have $\check{S}_{\mu}\left(A^{\prime}\right)=C_{\mu}(4,6,0)-C_{\mu}(0,0,3)=4 \mu(\{\mathrm{M}, \mathrm{S}\})+2 \mu(\{\mathrm{S}\})-3 \mu(\{\mathrm{L}\})$ and $\check{S}_{\mu}\left(B^{\prime}\right)=4 \mu(\{\mathrm{M}, \mathrm{S}\})+\mu(\{\mathrm{S}\})-2 \mu(\{\mathrm{L}\})$. Hence $(6)$ is equivalent to

$$
\mu(\{\mathrm{S}\})<\mu(\{\mathrm{L}\}) .
$$

Similarly, relation (7) is equivalent to

$$
\mu(\{\mathrm{S}\})>\mu(\{\mathrm{L}\})
$$

which contradicts previous inequality. Henceforth, the Šipoš integral is not able to model both (6) and (7).

Let us try to model (6) and (7) with the CPT model. We have $C P T\left(A^{\prime}\right)=$ $C_{\mu_{1}}(4,6,0)-C_{\mu_{2}}(0,0,3)=4 \mu_{1}(\{\mathrm{M}, \mathrm{S}\})+2 \mu_{1}(\{\mathrm{~S}\})-3 \mu_{2}(\{\mathrm{~L}\})$ and $C P T\left(B^{\prime}\right)=$ $4 \mu_{1}(\{\mathrm{M}, \mathrm{S}\})+\mu_{1}(\{\mathrm{~S}\})-2 \mu_{2}(\{\mathrm{~L}\})$. Hence $(6)$ is equivalent to

$$
\mu_{1}(\{\mathrm{~S}\})<\mu_{2}(\{\mathrm{~L}\}) .
$$


Similarly, relation (7) is equivalent to

$$
\mu_{1}(\{\mathrm{~S}\})>\mu_{2}(\{\mathrm{~L}\}) .
$$

Henceforth, the CPT model too fails to model both (6) and (7).

Let us try to model (6) and (7) with the extension of the Choquet integral to bi-capacities. We have $B C_{\mu}(4,6,-3)=C_{\nu_{\mu,\{\mathrm{M}, \mathrm{S}\}}}(4,6,3)=3 \nu_{\mu,\{\mathrm{M}, \mathrm{S}\}}(\{\mathrm{M}, \mathrm{S}, \mathrm{L}\})+$ $\nu_{\mu,\{\mathrm{M}, \mathrm{S}\}}(\{\mathrm{M}, \mathrm{S}\})+2 \nu_{\mu,\{\mathrm{M}, \mathrm{S}\}}(\{\mathrm{S}\})=3 \mu(\{\mathrm{M}, \mathrm{S}\},\{\mathrm{L}\})+\mu(\{\mathrm{M}, \mathrm{S}\}, \emptyset)+2 \mu(\{\mathrm{S}\}, \emptyset)$ and $B C_{\mu}(4,5,-2)=2 \mu(\{\mathrm{M}, \mathrm{S}\},\{\mathrm{L}\})+2 \mu(\{\mathrm{M}, \mathrm{S}\}, \emptyset)+\mu(\{\mathrm{S}\}, \emptyset)$. Hence (6) is equivalent to

$$
\mu(\{\mathrm{M}, \mathrm{S}\}, \emptyset)-\mu(\{\mathrm{M}, \mathrm{S}\},\{\mathrm{L}\})>\mu(\{\mathrm{S}\}, \emptyset)
$$

Similarly, relation (7) is equivalent to

$$
\mu(\{\mathrm{S}\},\{\mathrm{L}\})>0 .
$$

There is no contradiction between these two inequalities. $B C_{\mu}$ is thus able to model the example. Bi-capacities are able to model this kind of preference, where the weight of one attribute compared to another one depends on a third attribute being in one of the ranges 'good' or 'bad'. More generally, bicapacities can model general preferences that are conditional on the nature of the attributes levels being good or bad. Such general preferences are often encountered in real applications.

\section{Axiomatic construction of the generalized Choquet integral}

In this section, we describe in details the construction of a MCDM model based on the generalized Choquet integral, and in particular axioms characterizing it. We follow the methodology recalled at the end of Section 2.3 for unipolar scale. For bipolar scales, we take as $\mathbf{0}_{i}$ the neutral level of $X_{i}$, as defined in Section 2.2. The $\mathbf{1}_{i}$ element keeps the same meaning as for unipolar scales (see the end of Section 2.3). On top of defining for each attribute $X_{i}$ the elements $\mathbf{0}_{i}$ and $\mathbf{1}_{i}$, we assume furthermore that there exists an element denoted by $-\mathbf{1}_{i}$ that is considered as bad and unsatisfactory. It is symmetric to the level $\mathbf{1}_{i}$. All levels have the same absolute meaning, so we impose:

$$
\begin{aligned}
& u_{1}\left(-\mathbf{1}_{1}\right)=\cdots=u_{n}\left(-\mathbf{1}_{n}\right) \\
& u_{1}\left(\mathbf{0}_{1}\right)=\cdots=u_{n}\left(\mathbf{0}_{n}\right) \\
& u_{1}\left(\mathbf{1}_{1}\right)=\cdots=u_{n}\left(\mathbf{1}_{n}\right)
\end{aligned}
$$


We make the assumption that the absolute levels $\mathbf{1}_{i}$ and $-\mathbf{1}_{i}$ exist on all scales. The case when some attributes lack the existence of one of the two levels $\mathbf{1}_{i}$ or $-\mathbf{1}_{i}$ is discussed in Section 5.1.

The structure of this section is the following. First, we define the kind of information we need from the DM in order to build the model (Section 4.1), namely intra-criterion and inter-criteria information, based on the absolute levels $-\mathbf{1}_{i}, \mathbf{0}_{i}$ and $\mathbf{1}_{i}$, which lead respectively to the utility functions and the bi-capacity. Second, we give conditions so that the utility functions $u_{i}$, $i=1, \ldots, n$ and the bi-capacity are ratio scales (Section 4.2). Following that, we derive requirements on $F, \mu$ and the $u_{i}$ 's from the intra-criterion and inter-criteria conditions (Section 4.3). Finally, we propose a set of axioms (Section 4.4) from which the general Choquet integral follows (Section 4.5). Some properties of this general Choquet integral are presented (Section 4.6).

\subsection{Required information}

We describe first intra-criterion information.

We consider here the mapping (denoted by $u_{i}$ ) from the attribute $X_{i}$ to the satisfaction scale $\mathbb{R}$. Unlike the case of interval scales [23], we do not construct $u_{i}$ in one single step. The positive part $X_{i}^{+}:=\left\{x_{i} \in X_{i}, x_{i} \succeq_{i} \mathbf{0}_{i}\right\}$ and negative part $X_{i}^{-}:=\left\{x_{i} \in X_{i}, x_{i} \preceq_{i} \mathbf{0}_{i}\right\}$ of $X_{i}$ are dealt with separately. As a consequence, we introduce the two subsets $X\rfloor_{i}^{+}$and $\left.X\right\rfloor_{i}^{-}($for $i \in N)$ of $X$ defined by

$$
\left.X\rfloor_{i}^{+}:=\left\{\left(x_{i}, \mathbf{0}_{-i}\right), x_{i} \in X_{i}^{+}\right\}, X\right\rfloor_{i}^{-}:=\left\{\left(x_{i}, \mathbf{0}_{-i}\right), x_{i} \in X_{i}^{-}\right\} .
$$

We ask the DM not only the ranking of the elements of $X\rfloor_{i}^{+}$and $\left.X\right\rfloor_{i}^{-}$but also the difference of satisfaction degree between pairs of elements of $X\rfloor_{i}^{+}$and $X\rfloor_{i}^{-}$. The interval scale constructed on $\left.X\right\rfloor_{i}^{+}$(respectively $\left.X\right\rfloor_{i}^{-}$) is denoted by $u_{i}^{+}$(resp. $\left.u_{i}^{-}\right) \cdot u_{i}^{+}$and $u_{i}^{-}$are defined by :

$\left(\right.$ Intra $\left._{a}\right)$ For $\epsilon \in\{-,+\}$, and for all $x_{i}, y_{i} \in X_{i}^{\epsilon}, u_{i}^{\epsilon}\left(x_{i}\right) \geq u_{i}^{\epsilon}\left(y_{i}\right) \Leftrightarrow\left(x_{i}, \mathbf{0}_{-i}\right) \succeq$ $\left(y_{i}, \mathbf{0}_{-i}\right)$.

(Intra ${ }_{b}$ ) For $\epsilon \in\{-,+\}$, and for all $x_{i}, y_{i}, z_{i}, w_{i} \in X_{i}^{\epsilon}$ such that $u_{i}^{\epsilon}\left(x_{i}\right)>$ $u_{i}^{\epsilon}\left(y_{i}\right)$ and $u_{i}^{\epsilon}\left(w_{i}\right)>u_{i}^{\epsilon}\left(z_{i}\right)$, we have

$$
\frac{u_{i}^{\epsilon}\left(x_{i}\right)-u_{i}^{\epsilon}\left(y_{i}\right)}{u_{i}^{\epsilon}\left(w_{i}\right)-u_{i}^{\epsilon}\left(z_{i}\right)}=: k^{\epsilon}\left(x_{i}, y_{i}, w_{i}, z_{i}\right)>0
$$

if and only if the difference of satisfaction degree that the DM feels between $\left(x_{i}, \mathbf{0}_{-i}\right)$ and $\left(y_{i}, \mathbf{0}_{-i}\right)$ is $k^{\epsilon}\left(x_{i}, y_{i}, w_{i}, z_{i}\right)$ times as large as the difference of satisfaction between $\left(w_{i}, \mathbf{0}_{-i}\right)$ and $\left(z_{i}, \mathbf{0}_{-i}\right)$. 
$\left(\operatorname{Intra}_{c}\right) u_{i}^{+}\left(\mathbf{0}_{i}\right)=0, u_{i}^{+}\left(\mathbf{1}_{i}\right)=1, u_{i}^{-}\left(\mathbf{0}_{i}\right)=0$ and $u_{i}^{-}\left(-\mathbf{1}_{i}\right)=-1$.

Conditions $u_{i}^{+}\left(\mathbf{0}_{i}\right)=0$ and $u_{i}^{-}\left(\mathbf{0}_{i}\right)=0$ ensure that the two scales $u_{i}^{+}$and $u_{i}^{-}$ match at the element $\mathbf{0}_{i}$.

In order to be able to construct two unique scales $u_{i}^{+}$and $u_{i}^{-}$from $\left(\right.$ Intra $\left._{a}\right),\left(\right.$ Intra $\left._{b}\right)$ and (Intra $)$, some consistency assumptions shall be made :

$\left(\right.$ Intra $_{d}$ ) For $\epsilon \in\{-,+\}$, and for all $x_{i}, y_{i}, w_{i}, z_{i}, r_{i}, s_{i} \in X_{i}^{\epsilon}$ such that $u_{i}^{\epsilon}\left(x_{i}\right)>u_{i}^{\epsilon}\left(y_{i}\right), u_{i}^{\epsilon}\left(w_{i}\right)>u_{i}^{\epsilon}\left(z_{i}\right)$ and $u_{i}^{\epsilon}\left(r_{i}\right)>u_{i}^{\epsilon}\left(s_{i}\right)$,

$$
k^{\epsilon}\left(x_{i}, y_{i}, w_{i}, z_{i}\right) \times k^{\epsilon}\left(w_{i}, z_{i}, r_{i}, s_{i}\right)=k^{\epsilon}\left(x_{i}, y_{i}, r_{i}, s_{i}\right) .
$$

Assumption (Intra $\mathbf{I}_{c}$ ) implies that functions $u_{i}^{+}$and $u_{i}^{-}$cannot be constant. In particular, one has $u_{i}^{+}\left(\mathbf{1}_{i}\right) \neq u_{i}^{+}\left(\mathbf{0}_{i}\right)$ and $u_{i}^{-}\left(-\mathbf{1}_{i}\right) \neq u_{i}^{-}\left(\mathbf{0}_{i}\right)$. By (Intra $\left.{ }_{a}\right)$, this gives

$$
\left(\mathbf{1}_{i}, \mathbf{0}_{-i}\right) \not\left(\mathbf{0}_{N}\right),\left(-\mathbf{1}_{i}, \mathbf{0}_{-i}\right) \not\left(\mathbf{0}_{N}\right) .
$$

These conditions are essential for the construction of the utility functions from $X\rfloor_{i}^{+}$and $\left.X\right\rfloor_{i}^{-}$.

The scale $u_{i}$ is then defined by

$$
u_{i}\left(x_{i}\right)= \begin{cases}u_{i}^{+}\left(x_{i}\right) & \text { if } x_{i} \in X_{i}^{+} \\ u_{i}^{-}\left(x_{i}\right) & \text { otherwise }\end{cases}
$$

We give now inter-criteria information.

The following subset of $X$ depicts a variety of alternatives that mix satisfactory elements with unsatisfactory ones :

$$
X\rceil_{\{-1,0,1\}}:=\left\{\left(\mathbf{1}_{A},-\mathbf{1}_{A^{\prime}}, \mathbf{0}_{-A \cup A^{\prime}}\right),\left(A, A^{\prime}\right) \in \mathcal{Q}(N)\right\} .
$$

The satisfaction degree of $\left(\mathbf{1}_{A},-\mathbf{1}_{A^{\prime}}, \mathbf{0}_{-A \cup A^{\prime}}\right)$ will be denoted by $\mu\left(A, A^{\prime}\right)$. Unlike the case of $X\rceil_{\{0,1\}}:=\left\{\left(\mathbf{1}_{A}, \mathbf{0}_{-A}\right)\right\}$ used in the unipolar case, there are now three special points whose value must be fixed: $\mu(\emptyset, \emptyset)=0, \mu(N, \emptyset)=1$ and $\mu(\emptyset, N)=-1$. These conditions are rather natural since $\mu(\emptyset, \emptyset)=0$ means that the act which is neutral on all attributes is also neutral, $\mu(N, \emptyset)=$ 1 means that the act which is satisfactory on all attributes is also satisfactory, and $\mu(\emptyset, N)=-1$ means that the act which is unsatisfactory on all attributes is also unsatisfactory. Since there are only two degrees of freedom on an interval scale, one of these three points must be removed from the scale. We decide to remove $(\emptyset, N)$. Let $\mathcal{Q}^{*}(N):=\mathcal{Q}(N) \backslash\{(\emptyset, N)\}$. As before, we ask information from which one can obtain a satisfaction scale defined on $X\rceil_{\{-1,0,1\}}$. Let $\mu$ be defined on $\mathcal{Q}^{*}(N)$ by 
$\left(\right.$ Inter $\left._{a}\right) \forall\left(A, A^{\prime}\right),\left(B, B^{\prime}\right) \in \mathcal{Q}^{*}(N), \mu\left(A, A^{\prime}\right) \geq \mu\left(B, B^{\prime}\right) \Leftrightarrow\left(\mathbf{1}_{A},-\mathbf{1}_{A^{\prime}}, \mathbf{0}_{-A \cup A^{\prime}}\right) \succeq$ $\left(\mathbf{1}_{B},-\mathbf{1}_{B^{\prime}}, \mathbf{0}_{-B \cup B^{\prime}}\right)$.

(Inter $\left.{ }_{b}\right) \forall\left(A, A^{\prime}\right),\left(B, B^{\prime}\right),\left(C, C^{\prime}\right),\left(D, D^{\prime}\right) \in \mathcal{Q}^{*}(N)$ such that $\mu\left(A, A^{\prime}\right)>$ $\mu\left(B, B^{\prime}\right)$ and $\mu\left(C, C^{\prime}\right)>\mu\left(D, D^{\prime}\right)$, we have

$$
\frac{\mu\left(A, A^{\prime}\right)-\mu\left(B, B^{\prime}\right)}{\mu\left(C, C^{\prime}\right)-\mu\left(D, D^{\prime}\right)}=: k\left(A, A^{\prime}, B, B^{\prime}, C, C^{\prime}, D, D^{\prime}\right)>0
$$

if and only if the difference of satisfaction degree that the DM feels between $\left(\mathbf{1}_{A},-\mathbf{1}_{A^{\prime}}, \mathbf{0}_{-A \cup A^{\prime}}\right)$ and $\left(\mathbf{1}_{B},-\mathbf{1}_{B^{\prime}}, \mathbf{0}_{-B \cup B^{\prime}}\right)$ is $k\left(A, A^{\prime}, B, B^{\prime}, C, C^{\prime}, D, D^{\prime}\right)$ times as large as the difference of satisfaction between $\left(\mathbf{1}_{C},-\mathbf{1}_{C^{\prime}}, \mathbf{0}_{-C \cup C^{\prime}}\right)$ and $\left(\mathbf{1}_{D},-\mathbf{1}_{D^{\prime}}, \mathbf{0}_{-D \cup D^{\prime}}\right)$.

$\left(\right.$ Inter $\left._{c}\right) \mu(\emptyset, \emptyset)=0, \mu(N, \emptyset)=1$ and $\forall\left(A, A^{\prime}\right) \in \mathcal{Q}^{*}(N), \mu\left(A, A^{\prime}\right) \in[-1,1]$.

$\mu$ defined by (Inter ${ }_{a}$ ) and (Inter ${ }_{b}$ ) corresponds to an interval scale. Condition $\left(\operatorname{Inter}_{c}\right.$ ) fixes these two degrees of freedom. Henceforth, $\mu$ is uniquely determined by $\left(\right.$ Inter $\left._{a}\right),\left(\right.$ Inter $\left._{b}\right)$ and $\left(\right.$ Inter $\left._{c}\right)$. One can now define $\mu$ on $\mathcal{Q}(N)$ as follows :

$$
\mu\left(A, A^{\prime}\right)= \begin{cases}\mu\left(A, A^{\prime}\right) & \text { if }\left(A, A^{\prime}\right) \in \mathcal{Q}^{*}(N) \\ -1 & \text { if }\left(A, A^{\prime}\right)=(\emptyset, N)\end{cases}
$$

In order to be able to construct a unique scale from (Inter $\left.{ }_{a}\right),\left(\right.$ Inter $\left._{b}\right)$ and $\left(\operatorname{Inter}_{c}\right)$, some consistency assumptions shall be made :

(Inter $\left._{d}\right) \forall A, A^{\prime}, B, B^{\prime}, C, C^{\prime}, D, D^{\prime}, E, E^{\prime}, F, F^{\prime} \subset N$ such that $\mu\left(A, A^{\prime}\right)>$ $\mu\left(B, B^{\prime}\right), \mu\left(C, C^{\prime}\right)>\mu\left(D, D^{\prime}\right)$ and $\mu\left(E, E^{\prime}\right)>\mu\left(F, F^{\prime}\right)$

$$
k\left(A, A^{\prime}, B, B^{\prime}, C, C^{\prime}, D, D^{\prime}\right) \times k\left(C, C^{\prime}, D, D^{\prime}, E, E^{\prime}, F, F^{\prime}\right)=k\left(A, A^{\prime}, B, B^{\prime}, E, E^{\prime}, F, F^{\prime}\right) .
$$

For $A \subset A^{\prime}$, the preference relation $\succeq$ should satisfy $\left(\mathbf{1}_{A},-\mathbf{1}_{B}, \mathbf{0}_{-A \cup B}\right) \preceq$ $\left(\mathbf{1}_{A^{\prime}},-\mathbf{1}_{B}, \mathbf{0}_{-A^{\prime} \cup B}\right)$, so that one should have $\mu(A, B) \leq \mu\left(A^{\prime}, B\right)$. Similarly, for $B \subset B^{\prime}$, it is natural to have $\left(\mathbf{1}_{A},-\mathbf{1}_{B}, \mathbf{0}_{-A \cup B}\right) \succeq\left(\mathbf{1}_{A},-\mathbf{1}_{B^{\prime}}, \mathbf{0}_{-A \cup B^{\prime}}\right)$, and thus $\mu(A, B) \geq \mu\left(A, B^{\prime}\right)$. Previous two conditions on $\mu$ are the monotonicity conditions on $\mu$.

From above arguments, $\mu$ defined as previously is a bi-capacity.

Let us reflect on the last condition in (Inter $\left.{ }_{c}\right)$. Since $(\emptyset, N)$ is removed from $\mathcal{Q}^{*}(N)$, nothing ensures the monotonicity of $\mu$ defined on $\mathcal{Q}(N)$. Since $\mu(\emptyset, N)=-1$, condition $\mu\left(A, A^{\prime}\right) \in[-1,1]$ for $\left(A, A^{\prime}\right) \in \mathcal{Q}^{*}(N)$ is necessary and sufficient to obtain that $\mu$ defined on $\mathcal{Q}(N)$ is monotonic. 


\subsection{Measurement conditions}

The element $\mathbf{0}_{i}$ separates the levels of attractiveness from those representing repulsiveness. The absolute meaning of the $\mathbf{0}_{i}$ s elements deters us from shifting the scale. However, when only elements of the $X_{i}^{+}$s are considered, the real meaning of $\mathbf{0}_{i}$ is lost and thus this latter can be shifted. In other words, when we restrict ourself to positive values, $u_{i}^{+}$can be changed into $\alpha u_{i}^{+}+\beta$, with $\alpha>0$ and $\beta \geq 0$. The same holds when only negative values are considered (with $\alpha>0$ and $\beta \leq 0$ ).

By commensurateness arguments, one must change all $u_{i} \mathrm{~s}$ in $\alpha u_{i}+\beta$ with the same $\alpha$ and $\beta$ (one cannot change only one scale) when all arguments are above the zero level (see the justification of (SPLC)). Let $X^{+}:=X_{1}^{+} \times \cdots \times$ $X_{n}^{+}$and $X^{-}:=X_{1}^{-} \times \cdots \times X_{n}^{-}$. The measurement conditions for interval scales imply thus that the ratios $\frac{u(x)-u(y)}{u(z)-u(t)}$ for all $x, y, z, t \in X^{+}$shall not be changed if all the $u_{i}$ s are changed into $\alpha u_{i}+\beta$ with $\alpha>0$ and $\beta \geq 0$. We assume in this paper that this property holds only for the acts that we are considering in our construction, that is to say for $x, y, z, t \in X\rfloor_{i}^{+}$and $\left.x, y, z, t \in X\right\rceil_{\{-1,0,1\}} \cap X^{+}$. The same holds for the negative part. We have $\left.X\rceil_{\{0,1\}}=X\right\rceil_{\{-1,0,1\}} \cap X^{+}$ and $\left.X\rceil_{\{0,-1\}}:=X\right\rceil_{\{-1,0,1\}} \cap X^{-}=\left\{\left(-\mathbf{1}_{A}, \mathbf{0}_{-A}\right), A \subset N\right\}$. This leads to the following requirement :

$\left(\right.$ Intra $\left._{e}\right)$ The ratio $\frac{u(x)-u(y)}{u(z)-u(t)}$ for all $\left.\left.(x, y, z, t) \in \bigcup_{i \in N}(X\rfloor_{i}^{+}\right)^{4} \cup(X\rceil_{\{0,1\}}\right)^{4}$ (respectively $\left.\left.\left.\bigcup_{i \in N}(X\rfloor_{i}^{-}\right)^{4} \cup(X\rceil_{\{0,-1\}}\right)^{4}\right)$ shall not be changed if all the $u_{i}$ s are changed into $\alpha u_{i}+\beta$ with $\alpha>0$ and $\beta \geq 0$ (resp. $\beta \leq 0$ ).

This condition is no more than the requirement that the $u_{i}$ 's are commensurate interval scales on $X^{+}$and $X^{-}$.

In [23], we justified the fact that capacities correspond to ratio scales. The argument can be carried over to the case of bi-capacities. As a consequence, $\mu$ could be changed into $\gamma \mu$ with $\gamma \in \mathbb{R}^{+}$. As previously, the following requirement is imposed :

(Inter ${ }_{e}$ ) The ratio $\frac{u(x)-u(y)}{u(z)-u(t)}$ for all $\left.\left.\left.(x, y, z, t) \in(X\rfloor_{i}^{+}\right)^{4} \cup(X\rfloor_{i}^{-}\right)^{4} \cup(X\rceil_{\{0,1\}}\right)^{4}$ $\left.\cup(X\rceil_{\{0,-1\}}\right)^{4}$ shall not be changed if $\mu$ is changed into $\gamma \mu$, with $\gamma \in \mathbb{R}^{+}$.

\subsection{Conditions on the model}

It is now natural to write $u$ as follows:

$$
u(x)=F_{\mu}\left(u_{1}\left(x_{1}\right), \ldots, u_{n}\left(x_{n}\right)\right),
$$


where $F_{\mu}$ is the aggregation operator. $F_{\mu}$ depends on $\mu$ in a way that is not known for the moment. From now on, we assume that the preferences of the DM can be modeled by $u$ given by (9).

We study the conditions on $F_{\mu}$ implied by the previous requirements.

We consider first the consequences of intra-criterion information on the aggregation function $F_{\mu}$.

Lemma 3 If $u$ satisfies (9), and if conditions (Intra $\left.\mathbf{I}_{a}\right)$, (Intra $\left.\mathbf{I}_{b}\right)$, (Intra $\mathbf{I}_{c}$ ), $\left(\operatorname{Intra}_{d}\right),\left(\right.$ Intra $\left._{e}\right),\left(\right.$ Inter $\left._{a}\right),\left(\right.$ Inter $\left._{b}\right),\left(\right.$ Inter $\left._{c}\right),\left(\right.$ Inter $\left._{d}\right)$ and $\left(\right.$ Inter $\left._{e}\right)$ are fulfilled, then for all $\theta \in\{-1,1\}, \theta a_{i}, \theta b_{i}, \theta c_{i}, \theta d_{i} \geq 0$, and for all $\alpha>0$, $\theta \beta \geq 0, \gamma \in \mathbb{R}_{+}$,

$$
\frac{F_{\gamma \mu}\left(\alpha a_{i}+\beta, \beta_{-i}\right)-F_{\gamma \mu}\left(\alpha b_{i}+\beta, \beta_{-i}\right)}{F_{\gamma \mu}\left(\alpha c_{i}+\beta, \beta_{-i}\right)-F_{\gamma \mu}\left(\alpha d_{i}+\beta, \beta_{-i}\right)}=\frac{a_{i}-b_{i}}{c_{i}-d_{i}} .
$$

Proof : For $x_{i} \in X_{i}^{+}, u\left(x_{i}, \mathbf{0}_{-i}\right)$ and $u_{i}^{+}\left(x_{i}\right)$ correspond to two possible interval scales related to the same act $\left.\left(x_{i}, \mathbf{0}_{-i}\right) \in X\right\rfloor_{i}^{+}$. Henceforth, by $\left(\operatorname{Intra}_{a}\right),\left(\operatorname{Intra}_{b}\right)$, and $\left(\operatorname{Intra}_{d}\right)$

$$
\frac{u\left(x_{i}, \mathbf{0}_{-i}\right)-u\left(y_{i}, \mathbf{0}_{-i}\right)}{u\left(w_{i}, \mathbf{0}_{-i}\right)-u\left(z_{i}, \mathbf{0}_{-i}\right)}=\frac{u_{i}^{+}\left(x_{i}\right)-u_{i}^{+}\left(y_{i}\right)}{u_{i}^{+}\left(w_{i}\right)-u_{i}^{+}\left(z_{i}\right)} .
$$

All arguments $x_{i}, y_{i}, w_{i}, z_{i}$ and $\mathbf{0}_{-i}$ are above the zero level. We have

$$
\frac{F_{\mu}\left(u_{i}^{+}\left(x_{i}\right), u_{-i}^{+}\left(\mathbf{0}_{-i}\right)\right)-F_{\mu}\left(u_{i}^{+}\left(y_{i}\right), u_{-i}^{+}\left(\mathbf{0}_{-i}\right)\right)}{F_{\mu}\left(u_{i}^{+}\left(w_{i}\right), u_{-i}^{+}\left(\mathbf{0}_{-i}\right)\right)-F_{\mu}\left(u_{i}^{+}\left(z_{i}\right), u_{-i}^{+}\left(\mathbf{0}_{-i}\right)\right)}=\frac{u_{i}^{+}\left(x_{i}\right)-u_{i}^{+}\left(y_{i}\right)}{u_{i}^{+}\left(w_{i}\right)-u_{i}^{+}\left(z_{i}\right)} .
$$

Replacing $u_{i}^{+}$by $\alpha u_{i}^{+}+\beta \forall i \in N$ (see $\left(\right.$ Intra $\left.\left._{e}\right)\right)$ and $\mu$ by $\gamma \mu$ (see $\left(\right.$ Inter $\left._{e}\right)$ ), we obtain for $\alpha>0$ and $\beta \geq 0$

$$
\frac{F_{\gamma \mu}\left(\alpha u_{i}^{+}\left(x_{i}\right)+\beta, \beta_{-i}\right)-F_{\gamma \mu}\left(\alpha u_{i}^{+}\left(y_{i}\right)+\beta, \beta_{-i}\right)}{F_{\gamma \mu}\left(\alpha u_{i}^{+}\left(w_{i}\right)+\beta, \beta_{-i}\right)-F_{\gamma \mu}\left(\alpha u_{i}^{+}\left(z_{i}\right)+\beta, \beta_{-i}\right)}=\frac{u_{i}^{+}\left(x_{i}\right)-u_{i}^{+}\left(y_{i}\right)}{u_{i}^{+}\left(w_{i}\right)-u_{i}^{+}\left(z_{i}\right)} .
$$

Now, setting $a_{i}^{\prime}=u_{i}^{+}\left(x_{i}\right), b_{i}^{\prime}=u_{i}^{+}\left(y_{i}\right), c^{\prime}{ }_{i}=u_{i}^{+}\left(w_{i}\right)$ and $d_{i}^{\prime}=u_{i}^{+}\left(z_{i}\right)$, we have $a_{i}^{\prime}, b_{i}^{\prime}, c_{i}^{\prime}, d_{i}^{\prime} \geq 0$. Hence, we obtain for all $a_{i}^{\prime}, b_{i}^{\prime}, c_{i}^{\prime}, d^{\prime}{ }_{i} \in \operatorname{Range}\left(u_{i}^{+}\right)$, and for all $\alpha^{\prime}>0, \beta \geq 0$, and $\gamma \in \mathbb{R}_{+}$,

$$
\frac{F_{\gamma \mu}\left(\alpha^{\prime} a_{i}^{\prime}+\beta, \beta_{-i}\right)-F_{\gamma \mu}\left(\alpha^{\prime} b_{i}^{\prime}+\beta, \beta_{-i}\right)}{F_{\gamma \mu}\left(\alpha^{\prime} c^{\prime}{ }_{i}+\beta, \beta_{-i}\right)-F_{\gamma \mu}\left(\alpha^{\prime} d^{\prime}{ }_{i}+\beta, \beta_{-i}\right)}=\frac{a_{i}^{\prime}-b_{i}^{\prime}}{c_{i}^{\prime}-d_{i}^{\prime}} .
$$


For any $\delta>0$, set $\alpha=\frac{\alpha^{\prime}}{\delta}, a_{i}=\delta a^{\prime}{ }_{i}, b_{i}=\delta b^{\prime}{ }_{i}, c_{i}=\delta c^{\prime}{ }_{i}$ and $d_{i}=\delta d^{\prime}{ }_{i}$. The set of all possible values of $a_{i}$ when $\delta \in \mathbb{R}_{+}^{*}$ and $a^{\prime}{ }_{i} \in \operatorname{Range}\left(u_{i}^{+}\right)$is $\mathbb{R}_{+}$. Hence, for all $a_{i}, b_{i}, c_{i}, d_{i} \geq 0$, and for all $\alpha>0, \beta \geq 0$, and $\gamma \in \mathbb{R}_{+},(10)$ holds.

We obtain by a similar reasoning on $u_{i}^{-}$that for all $a_{i}, b_{i}, c_{i}, d_{i} \leq 0$, and for all $\alpha>0, \beta \leq 0$, and $\gamma \in \mathbb{R}_{+}$, equation (10) also holds.

We now give the consequences of inter-criteria information on the aggregation function $F_{\mu}$.

By conditions (Inter $\left.{ }_{a}\right)$ and $\left(\right.$ Inter $\left._{b}\right), u\left(\mathbf{1}_{A},-\mathbf{1}_{A^{\prime}}, \mathbf{0}_{-A \cup A^{\prime}}\right)$ and $\mu\left(A, A^{\prime}\right)$

correspond to two possible satisfaction scales related to the act $\left(\mathbf{1}_{A},-\mathbf{1}_{A^{\prime}}, \mathbf{0}_{-A \cup A^{\prime}}\right) \in$ $X\rceil_{\{-1,0,1\}}$. Hence

$$
\frac{u\left(\mathbf{1}_{A},-\mathbf{1}_{A^{\prime}}, \mathbf{0}_{-A \cup A^{\prime}}\right)-u\left(\mathbf{1}_{B},-\mathbf{1}_{B^{\prime}}, \mathbf{0}_{-B \cup B^{\prime}}\right)}{u\left(\mathbf{1}_{C},-\mathbf{1}_{C^{\prime}}, \mathbf{0}_{-C \cup C^{\prime}}\right)-u\left(\mathbf{1}_{D},-\mathbf{1}_{D^{\prime}}, \mathbf{0}_{-D \cup D^{\prime}}\right)}=\frac{\mu\left(A, A^{\prime}\right)-\mu\left(B, B^{\prime}\right)}{\mu\left(C, C^{\prime}\right)-\mu\left(D, D^{\prime}\right)},
$$

which gives

$$
\begin{gathered}
\frac{F_{\mu}\left(u_{A}\left(\mathbf{1}_{A}\right), u_{A^{\prime}}\left(-\mathbf{1}_{A^{\prime}}\right), u_{-A \cup A^{\prime}}\left(\mathbf{0}_{-A \cup A^{\prime}}\right)\right)-F_{\mu}\left(u_{B}\left(\mathbf{1}_{B}\right), u_{B^{\prime}}\left(-\mathbf{1}_{B^{\prime}}\right), u_{-B \cup B^{\prime}}\left(\mathbf{0}_{-B \cup B^{\prime}}\right)\right)}{F_{\mu}\left(u_{C}\left(\mathbf{1}_{C}\right), u_{C^{\prime}}\left(-\mathbf{1}_{C^{\prime}}\right), u_{-C \cup C^{\prime}}\left(\mathbf{0}_{-C \cup C^{\prime}}\right)\right)-F_{\mu}\left(u_{D}\left(\mathbf{1}_{D}\right), u_{D^{\prime}}\left(-\mathbf{1}_{D^{\prime}}\right), u_{-D \cup D^{\prime}}\left(\mathbf{0}_{-D \cup D^{\prime}}\right)\right)} \\
=\frac{\mu\left(A, A^{\prime}\right)-\mu\left(B, B^{\prime}\right)}{\mu\left(C, C^{\prime}\right)-\mu\left(D, D^{\prime}\right)}
\end{gathered}
$$

Lemma 4 If $u$ satisfies (9), and if conditions $\left(\operatorname{Intra}_{a}\right)$, (Intra $\left.{ }_{b}\right),\left(\right.$ Intra $\left._{c}\right)$, $\left(\right.$ Intra $\left._{d}\right),\left(\right.$ Intra $\left._{e}\right),\left(\right.$ Inter $\left._{a}\right),\left(\right.$ Inter $\left._{b}\right),\left(\operatorname{Inter}_{c}\right),\left(\right.$ Inter $\left._{d}\right)$ and $\left(\right.$ Inter $\left._{e}\right)$ are fulfilled, then for all $\alpha>0, \beta \geq 0, \gamma \in \mathbb{R}_{+}$

$$
\frac{F_{\gamma \mu}\left((\alpha+\beta)_{A}, \beta_{-A}\right)-F_{\gamma \mu}\left((\alpha+\beta)_{B}, \beta_{-B}\right)}{F_{\gamma \mu}\left((\alpha+\beta)_{C}, \beta_{-C}\right)-F_{\gamma \mu}\left((\alpha+\beta)_{D}, \beta_{-D}\right)}=\frac{\mu(A, \emptyset)-\mu(B, \emptyset)}{\mu(C, \emptyset)-\mu(D, \emptyset)}
$$

and for all $\alpha<0, \beta \leq 0, \gamma \in \mathbb{R}_{+}$,

$$
\frac{F_{\gamma \mu}\left((\alpha+\beta)_{A}, \beta_{-A}\right)-F_{\gamma \mu}\left((\alpha+\beta)_{B}, \beta_{-B}\right)}{F_{\gamma \mu}\left((\alpha+\beta)_{C}, \beta_{-C}\right)-F_{\gamma \mu}\left((\alpha+\beta)_{D}, \beta_{-D}\right)}=\frac{\mu(\emptyset, A)-\mu(\emptyset, B)}{\mu(\emptyset, C)-\mu(\emptyset, D)}
$$

Proof : Consider first $A^{\prime}=B^{\prime}=C^{\prime}=D^{\prime}=\emptyset$. By the measurement conditions given in section 4.2, one can change in (11) the utility functions $u_{i}$ by $\alpha u_{i}+\beta$ (with $\alpha>0$ and $\beta \geq 0$ ) when all arguments are above the zero level, and also change $\mu$ by $\gamma \mu\left(\gamma \in \mathbb{R}_{+}\right)$. This gives (12) for all $\alpha>0, \beta \geq 0$ and $\gamma \in \mathbb{R}_{+}$. We obtain similarly (13) for all $\alpha<0, \beta \leq 0$ and $\gamma \in \mathbb{R}_{+}$. 


\subsection{Axioms satisfied by the aggregation function}

The mapping $w \mapsto F_{\mu}(w)$ must be increasing, exactly as in (MC).

Monotonicity w.r.t. Bi-Capacities (MBC): For any $\mu \in$ $\mathcal{C}^{2}(N), \forall w, w^{\prime} \in \mathbb{R}^{n}$

$$
w_{i} \leq w_{i}^{\prime}, \forall i \in N \Rightarrow F_{\mu}(w) \leq F_{\mu}\left(w^{\prime}\right)
$$

Since $F_{\mu}$ aggregates commensurate scales and $F_{\mu}$ corresponds to a compensatory function and thus to some kind of average, it is natural to assume that whenever $\mu \in \mathcal{C}^{2}(N)$

$$
F_{\mu}(\beta, \ldots, \beta)=\beta, \forall \beta \geq 0 .
$$

We introduce the following axioms.

Properly Weighted w.r.t. Bi-Capacities (PWBC): For any $\mu \in \mathcal{C}^{2}(N), F_{\mu}\left(1_{A},-1_{A^{\prime}}, 0_{-A \cup A^{\prime}}\right)=\mu\left(A, A^{\prime}\right), \forall\left(A, A^{\prime}\right) \in \mathcal{Q}(N)$.

Stable under Positive Linear transformations with positive shifts for Bi-Capacities and binary acts $\left.\left(\mathrm{SPLBC}^{+}\right)\right)$:

For any $\mu \in \mathcal{C}^{2}(N)$ for all $A \subset N, \alpha>0$, and $\beta \geq 0$,

$$
F_{\mu}\left((\alpha+\beta)_{A}, \beta_{-A}\right)=\alpha F_{\mu}\left(1_{A}, 0_{-A}\right)+\beta
$$

Lemma 5 If $u$ satisfies (9), and if conditions (14), (MBC), (Intra $)_{a}$, $\left(\operatorname{Intra}_{b}\right),\left(\operatorname{Intra}_{c}\right),\left(\operatorname{Intra}_{d}\right),\left(\operatorname{Intra}_{e}\right),\left(\operatorname{Inter}_{a}\right),\left(\operatorname{Inter}_{b}\right),\left(\operatorname{Inter}_{c}\right),\left(\operatorname{Inter}_{d}\right)$ and $\left(\mathbf{I n t e r}_{e}\right)$ are fulfilled, then $(\mathbf{P W B C})$ and $\left(\mathbf{S P L B C}^{+}\right)$are satisfied.

Proof : Let $\mu$ follow from the construction of a DM's preferences based on intra-criterion and inter-criteria information. $\mu$ belongs to $\mathcal{C}^{2}(N)$ by the fundamental assumptions (MBC), (Inter $\left.)_{a}\right),\left(\right.$ Inter $\left._{c}\right)$. Applying (12), (14) and from Lemma 4 with $\gamma=1, B=D=\emptyset$ and $C=N$, we obtain

$$
F_{\mu}\left((\alpha+\beta)_{A}, \beta_{-A}\right)=\alpha \mu(A, \emptyset)+\beta, \forall \alpha>0, \forall \beta \geq 0
$$

By $\left(\operatorname{Intra}_{c}\right)$ and (11) with $B=B^{\prime}=D=D^{\prime}=C^{\prime}=\emptyset, C=N$,

$$
\frac{F_{\mu}\left(1_{A},-1_{A^{\prime}}, 0_{A \cup A^{\prime}}\right)-F_{\mu}\left(0_{N}\right)}{F_{\mu}\left(1_{N}\right)-F_{\mu}\left(0_{N}\right)}=\mu\left(A, A^{\prime}\right) .
$$


Hence, by (14), condition (PWBC) is satisfied for that $\mu$. Combining (15) and $(\mathbf{P W B C})$, we obtain $\left(\mathbf{S P L B C} \mathbf{C}^{+}\right)$for that $\mu$. Considering all potential decision strategies, we get (PWBC) and $\left(\mathbf{S P L B C}^{+}\right)$for all $\mu \in \mathcal{C}^{2}(N)$.

(PWBC) is a generalization of (PWC) to ternary acts. The justification of (PWBC) follows that of (PWC). The weight $\mu(A, B)$ corresponds to the overall evaluation of the ternary act that is satisfactory on $A$, unsatisfactory on $B$ and neutral on the other criteria. Moreover, $\left(\mathbf{S P L B C} \mathbf{B}^{+}\right)$is similar to $\left(\mathrm{SPLC}^{+}\right)$.

Bipolar aggregation quantifies a possible switch of behavior between attractive and repulsive values. The information related to these sign-dependent decision strategies is described by the bi-capacity $\mu . F_{\mu}(w)$ is thus some kind of interpolation of the weight $\mu$ at point $w$ and it holds in itself nothing special on the positive part compared to the negative one. $F$ just reproduces what $\mu$ tells. This implies that if we apply some symmetry through $\Phi$ (see equation (5)), it is similar to an exchange of the + and the - parts on the bipolar axis. For instance, if we switch the + and the - parts on all criteria, an act $w$ is transformed into $-w$ whereas a bi-game $\mu$ is transformed into $\mu^{\prime}(A, B)=\mu(B, A)$. The evaluation $F_{\mu}(w)$ of $w$ from $\mu$ shall be the same as the evaluation $F_{\mu^{\prime}}(-w)$ of $-w$ from $\mu^{\prime}$ if the scales are inverted. More generally, one can apply a symmetry only on a subset $A$ of criteria. For $A \subset N$, consider the following mapping $\Pi_{A}: \mathbb{R}^{n} \rightarrow \mathbb{R}^{n}$ defined by

$$
\left(\Pi_{A}(w)\right)_{i}= \begin{cases}w_{i} & \text { if } i \in A \\ -w_{i} & \text { otherwise }\end{cases}
$$

By (PWBC), $\mu\left(B, B^{\prime}\right)$ corresponds to the point $\left(1_{B},-1_{B^{\prime}}, 0_{-B \cup B^{\prime}}\right)$. Define $\Pi_{A} \circ \mu$ by $\Pi_{A} \circ \mu\left(B, B^{\prime}\right):=\mu\left(\Phi^{-1} \circ \Pi_{A} \circ \Phi\left(B, B^{\prime}\right)\right)$. We have

$$
\Pi_{A} \circ \Phi\left(B, B^{\prime}\right)=\left(1_{(B \cap A) \cup\left(B^{\prime} \backslash A\right)},-1_{(B \backslash A) \cup\left(B^{\prime} \cap A\right)}, 0_{-B \cup B^{\prime}}\right) .
$$

Hence

$$
\Pi_{A} \circ \mu\left(B, B^{\prime}\right)=\mu\left((B \cap A) \cup\left(B^{\prime} \backslash A\right),(B \backslash A) \cup\left(B^{\prime} \cap A\right)\right) .
$$

By symmetry arguments, it is reasonable to have $F_{\Pi_{A} \circ \mu}\left(\Pi_{A}(w)\right)$ being equal to $F_{\mu}(w)$.

Symmetry (Sym): For any $\mu \in \mathcal{G}^{2}(N)$, we have for all $A \subset N$

$$
F_{\mu}(w)=F_{\Pi_{A} \circ \mu}\left(\Pi_{A}(w)\right) .
$$


Equation (15) yields linearity of the mapping $\mu \mapsto F_{\mu}(w)$ for the acts of the form $w=\left((\alpha+\beta)_{A}, \beta_{-A}\right)$. More generally, the argument that motivates (LC) can be reproduced here, leading to the linearity of $F_{\mu}$ w.r.t. $\mu$. Bicapacities can be indeed decomposed as linear combinations of elementary bi-capacities. One can mention for instance the decomposition in terms of bi-unanimity games and the Möbius transform [17].

$F_{\mu}(w)$ is some kind of interpolation of $\mu$ at point $w$. Yet, $\mu$ contains information regarding decision strategies in all domains $\Sigma_{A}$ for $A \subset N$. Therefore, if $w_{i} \geq 0$, one should consider only the decision strategies related to nonnegative values on criterion $i$, hence for terms $\mu\left(B, B^{\prime}\right)$ of the bi-capacity for which $i$ does not belong to the negative part (i.e. $i \notin B^{\prime}$ ). Similarly, the only decision strategies that should be used for an act which score w.r.t. criterion $i$ is non-positive are the ones in the negative part of criterion $i$, that is depicted by $\mu\left(B, B^{\prime}\right)$ with $i \notin B$. The decision strategies used to determine the overall assessment of an element $w \in \Sigma_{A}, A \subset N$, are encoded by the terms of $\mu$ restricted to $\Phi^{-1}\left(\Delta_{A}\right)$, with

$$
\Phi^{-1}\left(\Delta_{\Sigma_{A}}\right)=\left\{\left(B, B^{\prime}\right) \in \mathcal{Q}(N), B \subset A, B^{\prime} \cap A=\emptyset\right\}
$$

Hence, linearity of $F_{\mu}(w)$ w.r.t. $\mu$ should hold in $\Phi^{-1}\left(\Delta_{A}\right)$ for $w \in \Sigma_{A}$.

Linearity w.r.t. Bi-Capacities (LBC): Let $A \subset N$. If $\mu, \mu_{1}, \ldots, \mu_{p} \in \mathcal{G}^{2}(N)$ satisfy $\mu\left(B, B^{\prime}\right)=\sum_{i=1}^{p} \alpha_{i} \mu_{i}\left(B, B^{\prime}\right)$ with $\alpha_{1}, \ldots, \alpha_{p} \in \mathbb{R}$, for all $\left(B, B^{\prime}\right) \in \Phi^{-1}\left(\Delta_{A}\right)$, then for all $w \in \Sigma_{A}$

$$
F_{\mu}(w)=\sum_{i=1}^{p} \alpha_{i} F_{\mu_{i}}(w)
$$

\subsection{Expression of the aggregation function}

Theorem $3\left\{F_{\mu}\right\}_{\mu}$ satisfies (LBC), (MBC), (PWBC), $\left(\mathrm{SPLBC}^{+}\right)$and (Sym) if and only if for any $\mu \in \mathcal{C}^{2}(N)$, and for any $w \in \mathbb{R}^{n}$,

$$
F_{\mu}(w)=B C_{\mu}(w)
$$

\section{Proof :}


- $\Leftarrow$ : Let $A \subset N$, and $w \in \Sigma_{A}$. We have $\Pi_{A}(w) \in \mathbb{R}_{+}^{n}$. Let $\tau$ be an ordering of $|w|:\left|w_{\tau(1)}\right| \leq \cdots \leq\left|w_{\tau(n)}\right|$. We write for $\nu \in \mathcal{C}^{2}(N)$

$$
\begin{aligned}
C_{\nu_{\mu, A}} & \left(\Pi_{A}(w)\right)=C_{\nu_{\mu, A}}(|w|) \\
= & \sum_{i=1}^{n}\left|w_{\tau(i)}\right|\left[\nu_{\mu, A}(\{\tau(i), \ldots, \tau(n)\})-\nu_{\mu, A}(\{\tau(i+1), \ldots, \tau(n)\})\right] .
\end{aligned}
$$

For $i \in N$, we have the following alternative. If $\tau(i) \in A$, then $w_{\tau(i)} \geq 0$ and thus, by monotonicity of $\mu$ (condition (i)), $\nu_{\mu, A}(\tau(i), \ldots, \tau(n)) \geq$ $\nu_{\mu, A}(\tau(i+1), \ldots, \tau(n))$. Hence $w_{\tau(i)} \mapsto F_{\mu}(w)$ is increasing. Now, if $\tau(i) \notin A$, then $w_{\tau(i)} \leq 0$ and thus, by monotonicity of $\mu$ (condition (ii)), $\nu_{\mu, A}(\tau(i), \ldots, \tau(n)) \leq \nu_{\mu, A}(\tau(i+1), \ldots, \tau(n))$. We deduce once more that $w_{\tau(i)} \mapsto F_{\mu}(w)$ is increasing. Hence axiom (MBC) is fulfilled.

For $\nu \in \mathcal{C}^{2}(N)$, We have for $\alpha>0$ and $\beta \geq 0$

$$
\begin{aligned}
& F_{\mu}\left((\alpha+\beta)_{A}, \beta_{-A}\right)=C_{\nu_{\mu, A}}\left((\alpha+\beta)_{A}, \beta_{-A}\right) \\
& \quad=\alpha C_{\nu_{\mu, A}}\left(1_{A}, 0_{-A}\right)+\beta \nu_{\mu, A}(N)=\alpha F_{\mu}\left(1_{A}, 0_{-A}\right)+\beta
\end{aligned}
$$

so that $\left(\mathrm{SPLBC}^{+}\right)$is satisfied.

Let $A, B \subset N, \mu \in \mathcal{G}^{2}(N)$ and $w \in \Sigma_{B}$. Let us check that $F_{\mu}(w)=$ $F_{\Pi_{A} \circ \mu}\left(\Pi_{A}(w)\right)$. On one hand,

$$
F_{\mu}(w)=C_{\nu_{\mu, B}}\left(\Pi_{B}(w)\right),
$$

where $\nu_{\mu, B}(C)=\mu(C \cap B, C \backslash B)$. On the other hand, $\Pi_{A}(w) \in \Sigma_{D}$ with $D=(B \cap A) \cup\left(B^{c} \cap A^{c}\right)$. Hence $\Pi_{D}\left(\Pi_{A}(w)\right) \in \Sigma_{N}$ and

$$
F_{\Pi_{A} \mathrm{o} \mu}\left(\Pi_{A}(w)\right)=C_{\nu^{\prime}}\left(\Pi_{D}\left(\Pi_{A}(w)\right)\right)
$$

where $\nu^{\prime}=\nu_{\Pi_{A} \circ \mu, D}$ is given by

$$
\begin{aligned}
\nu^{\prime}(C) & =\Pi_{A} \circ \mu(C \cap D, C \backslash D) \\
& =\mu[(C \cap D \cap A) \cup((C \backslash D) \backslash A),((C \cap D) \backslash A) \cup((C \backslash D) \cap A)] .
\end{aligned}
$$

We have

$$
\begin{aligned}
C \cap D \cap A & =C \cap A \cap\left[(B \cap A) \cup\left(B^{c} \cap A^{c}\right)\right] \\
& =[C \cap A \cap(B \cap A)] \cup \underbrace{\left[C \cap A \cap B^{c} \cap A^{c}\right]}_{=\emptyset} \\
& =C \cap B \cap A
\end{aligned}
$$


and

$$
\begin{aligned}
(C \cap D) \backslash A & =\underbrace{[(C \cap B \cap A) \backslash A]}_{=\emptyset} \cup\left[\left(C \cap B^{c} \cap A^{c}\right) \backslash A\right] \\
& =C \cap B^{c} \cap A^{c} .
\end{aligned}
$$

From the relation

$$
\begin{aligned}
C \backslash D & =C \backslash\left((B \cap A) \cup\left(B^{c} \cap A^{c}\right)\right) \\
& =(C \backslash(B \cap A)) \cap\left(C \backslash\left(B^{c} \cap A^{c}\right)\right) \\
& =(C \cap(A \backslash B)) \cup(C \cap(B \backslash A))
\end{aligned}
$$

one can deduce that

$$
\begin{aligned}
(C \backslash D) \backslash A & =\underbrace{[(C \cap(A \backslash B)) \backslash A]}_{=\emptyset} \cup[(C \cap(B \backslash A)) \backslash A] \\
& =C \cap(B \backslash A)
\end{aligned}
$$

and

$$
\begin{aligned}
(C \backslash D) \cap A & =(C \cap(A \backslash B) \cap A) \cup \underbrace{(C \cap(B \backslash A) \cap A)}_{=\emptyset} \\
& =C \cap(A \backslash B)
\end{aligned}
$$

Consequently

$$
\begin{aligned}
\nu^{\prime}(C) & =\mu\left[(C \cap B \cap A) \cup(C \cap(B \backslash A)),\left(C \cap B^{c} \cap A^{c}\right) \cup(C \cap(A \backslash B))\right] \\
& =\mu(C \cap B, C \backslash B)=\nu_{\mu, B}(C) .
\end{aligned}
$$

Moreover,

$$
\begin{aligned}
\Pi_{D}\left(\Pi_{A}(w)\right) & = \begin{cases}\left(\Pi_{A}(w)\right)_{i} & \text { if } i \in D \\
-\left(\Pi_{A}(w)\right)_{i} & \text { otherwise }\end{cases} \\
& = \begin{cases}w_{i} & \text { if } i \in(D \cap A) \cup\left(D^{c} \cap A^{c}\right) \\
-w_{i} & \text { otherwise }\end{cases}
\end{aligned}
$$

We have $D \cap A=B \cap A$. Furthermore, since $D^{c}=(B \backslash A) \cup(A \backslash B)$, it holds that $D^{c} \cap A^{c}=B \backslash A$. Consequently $(D \cap A) \cup\left(D^{c} \cap A^{c}\right)=B$, and

$$
\Pi_{D}\left(\Pi_{A}(w)\right)=\Pi_{B}(w)
$$


Previous relation combined with (16) gives

$$
F_{\Pi_{A} \circ \mu}\left(\Pi_{A}(w)\right)=C_{\nu^{\prime}}\left(\Pi_{D}\left(\Pi_{A}(w)\right)\right)=C_{\nu_{\mu, B}}\left(\Pi_{B}(w)\right)=F_{\mu}(w) .
$$

As a consequence, axiom (Sym) holds. It is easy to show that $F_{\mu}$ satisfies the other axioms and in particular (LBC).

- $\Rightarrow$ : Consider now $F_{\mu}$ satisfying (LBC), (MBC), (PWBC), (SPLBC $\left.{ }^{+}\right)$ and (Sym). Let $\mu \in \mathcal{C}^{2}(N)$ be fixed. For $\nu \in \mathcal{G}^{1}(N)$ define $\mu_{\nu} \in \mathcal{G}^{2}(N)$ by

$$
\mu_{\nu}\left(B, B^{\prime}\right)=\left\{\begin{array}{l}
\nu(B) \text { if } B^{\prime}=\emptyset \\
\min \left(\mu\left(B, B^{\prime}\right), \nu(B)\right) \text { if } B^{\prime} \neq \emptyset
\end{array}\right.
$$

Let $G_{\nu}$ defined in $\mathbb{R}_{+}^{n}$ such that

$$
G_{\nu}(w)=F_{\mu_{\nu}}(w)
$$

Let $\nu, \nu_{1}, \ldots, \nu_{p} \in \mathcal{G}^{1}(N)$ such that $\nu=\sum_{i=1}^{p} \alpha_{i} \nu_{i}$, with $\alpha_{1}, \ldots, \alpha_{p} \in$ $\mathbb{R}$. One has $\mu_{\nu}(B, \emptyset)=\sum_{i=1}^{p} \alpha_{i} \mu_{\nu_{i}}(B, \emptyset)$ for all $B \subset N$, where $\mu_{\nu}, \mu_{\nu_{1}}, \ldots, \mu_{\nu_{p}} \in \mathcal{G}^{2}(N)$. Then by (LBC), for all $w \in \mathbb{R}_{+}^{n}$

$$
G_{\nu}(w)=F_{\mu_{\nu}}(w)=\sum_{i=1}^{p} \alpha_{i} F_{\mu_{\nu_{i}}}(w)=\sum_{i=1}^{p} \alpha_{i} G_{\nu_{i}}(w)
$$

Hence $G_{\nu}$ satisfies $\left(\mathrm{LG}^{+}\right)$.

Consider $\nu \in \mathcal{C}^{1}(N)$. By construction, $\mu_{\nu}$ is monotone and normalized so that $\mu_{\nu} \in \mathcal{C}^{2}(N)$. Therefore, we have by (PWBC) for all $C \subset N$

$$
G_{\nu}\left(1_{C}, 0_{-C}\right)=F_{\mu_{\nu}}\left(1_{C}, 0_{-C}\right)=\mu_{\nu}(C, \emptyset)=\nu(C) .
$$

Hence $G_{\nu}$ satisfies (PWC).

Since $\mu_{\nu} \in \mathcal{C}^{2}(N)$, the mapping $w \mapsto F_{\Pi_{A} \circ \mu_{\nu}}(w)$ is increasing by (MBC). This shows that $\left(\mathbf{M C}^{+}\right)$is fulfilled.

By $\left(\mathrm{SPLBC}^{+}\right)$, and since $\mu_{\nu} \in \mathcal{C}^{2}(N)$, we have for any $C \subset N, \alpha>0$ and $\beta \geq 0$,

$$
\begin{aligned}
G_{\nu}\left((\alpha+\beta)_{C}, \beta_{-C}\right) & =F_{\mu_{\nu}}\left((\alpha+\beta)_{C}, \beta_{-C}\right)=\alpha F_{\mu_{\nu}}\left(1_{C}, 0_{-C}\right)+\beta \\
& =\alpha G_{\nu}\left(1_{C}, 0_{-C}\right)+\beta
\end{aligned}
$$


Hence $G_{\nu}$ satisfies $\left(\mathrm{SPLC}^{+}\right)$.

We conclude by Theorem 2 that $G_{\nu}$ is the Choquet integral w.r.t. $\nu$. We apply this to $\nu_{\mu, \emptyset}$ given by $\nu_{\mu, \emptyset}(B):=\mu(B, \emptyset)$. One has $\mu_{\nu_{\mu, \emptyset}}\left(B, B^{\prime}\right)=$ $\mu\left(B, B^{\prime}\right)$ for all $\left(B, B^{\prime}\right) \in \mathcal{Q}(N)$. We conclude that for all $w \in \mathbb{R}_{+}^{n}$,

$$
F_{\mu}(w)=F_{\mu_{\mu, \emptyset}}(w)=G_{\nu_{\mu, \emptyset}}(w)=C_{\nu_{\mu, \emptyset}}(w) .
$$

Consider now $\mu \in \mathcal{G}^{2}(N)$. Let $\nu \in \mathcal{G}^{1}(N)$ such that $\nu(B)=\mu(B, \emptyset)$. Using the Möbius decomposition, we write $\nu=\sum_{C \subset N} \alpha_{C} \nu_{C}$, where $\alpha_{C}$ are the Möbius coefficients, and $\nu_{C} \in \mathcal{C}^{1}(N)$ is the unanimity game w.r.t. $C$. Define $\mu_{C}$ by

$$
\mu_{C}\left(B, B^{\prime}\right)=\nu_{C}(B)-\nu_{C}\left(B^{\prime}\right) .
$$

Clearly, $\mu_{C} \in \mathcal{C}^{2}(N)$. Moreover, $\mu(B, \emptyset)=\sum_{C \subset N} \alpha_{C} \mu_{C}(B, \emptyset)$ for all $B \subset N$. By (LBC), for all $w \in \mathbb{R}_{+}^{n}$

$$
F_{\mu}(w)=\sum_{C \subset N} \alpha_{C} F_{\mu_{C}}(w)
$$

Since $\mu_{C} \in \mathcal{C}^{2}(N)$, one has $F_{\mu_{C}}(w)=C_{\nu_{C}}(w)$ by (17). Hence

$$
F_{\mu}(w)=\sum_{C \subset N} \alpha_{C} C_{\nu_{C}}(w)=C_{\sum_{C \subset N} \alpha_{C} \nu_{C}}(w)=C_{\nu}(w) .
$$

This proves that (17) holds also for $\mu \in \mathcal{G}^{2}(N)$.

Consider finally $\mu \in \mathcal{C}^{2}(N)$ and $A \subset N$. By (Sym), we have for $w \in \Sigma_{A}, F_{\mu}(w)=F_{\Pi_{A} \circ \mu}\left(\Pi_{A}(w)\right)$. Applying (17) to $\Pi_{A} \circ \mu \in \mathcal{G}^{2}(N)$, and $\Pi_{A}(w) \in \mathbb{R}_{+}^{n}$, we get $F_{\Pi_{A^{\circ} \mu}}\left(\Pi_{A}(x)\right)=C_{\nu_{\Pi_{A} \circ \mu \emptyset}}\left(\Pi_{A}(w)\right)$, where $\nu_{\Pi_{A} \circ \mu, \emptyset}(B)=\Pi_{A} \circ \mu(B, \emptyset)=\mu(B \cap A, B \backslash A)=\nu_{\mu, A}(B)$. Therefore $F_{\mu}(w)=C_{\nu_{\mu, A}}\left(\Pi_{A}(w)\right)$, so that $F_{\mu} \equiv B C_{\mu}$.

By (8) and (PWBC), the condition under which the utility functions can be constructed from $X\rfloor_{i}^{+}$and $\left.X\right\rfloor_{i}^{-}$is:

$$
\forall i \in N, \mu(\{i\}, \emptyset)>0 \text { and } \mu(\emptyset,\{i\})<0 .
$$

Combining Lemma 5 and Theorem 3, we get

Corollary 1 If $u$ satisfies (9), and if conditions (14), (LBC), (MBC), $\left(\mathbf{S y m}_{)}\left(\operatorname{Intra}_{a}\right),\left(\operatorname{Intra}_{b}\right),\left(\operatorname{Intra}_{c}\right),\left(\operatorname{Intra}_{d}\right),\left(\right.\right.$ Intra $\left._{e}\right),\left(\right.$ Inter $\left._{a}\right),\left(\right.$ Inter $\left._{b}\right)$, $\left(\right.$ Inter $\left._{c}\right),\left(\right.$ Inter $\left._{d}\right)$ and $\left(\right.$ Inter $\left._{e}\right)$ are fulfilled, then $\mu \in \mathcal{C}^{2}(N)$, and $F_{\mu} \equiv$ $\mathrm{BC}_{\mu}$. 


\subsection{Properties of bi-capacities}

In this section, we show that the aggregation operator $F_{\mu}$ defined on bicapacities (see Theorem 3) generalizes the Choquet integral, the Šipoš integral and the Cumulative Prospect Theory model.

Theorem 4 If $\mu$ satisfies $\mu(A, B)-\mu\left(A, B^{\prime}\right)=\mu\left(A^{\prime}, B\right)-\mu\left(A^{\prime}, B^{\prime}\right)$ for any $A, A^{\prime}, B, B^{\prime} \subset N$ with $(A, B),\left(A, B^{\prime}\right),\left(A^{\prime}, B\right),\left(A^{\prime}, B^{\prime}\right) \in \mathcal{Q}(N)$, then $F_{\mu}$ becomes the model of CPT.

Proof : It is easy to check that if $\mu$ satisfies $\mu(A, B)-\mu\left(A, B^{\prime}\right)=\mu\left(A^{\prime}, B\right)-$ $\mu\left(A^{\prime}, B^{\prime}\right)$, then there exist two capacities $\nu_{1}$ and $\nu_{2}$ such that $\mu(A, B)=$ $\nu_{1}(A)-\nu_{2}(B)$. Moreover, $w \mapsto C_{\nu_{1}}\left(w^{+}\right)-C_{\nu_{2}}\left(w^{-}\right)$satisfies the axioms (LBC), (MBC), (PWBC), (SPLBC $\left.{ }^{+}\right)$and (Sym). Hence, by uniqueness implied by Theorem 3 , one obtains $F_{\mu}(w)=C_{\nu_{1}}\left(w^{+}\right)-C_{\nu_{2}}\left(w^{-}\right)$.

Consider now the symmetric case.

Theorem 5 If $\mu$ satisfies $\mu(A, B)=-\mu(B, A)$ for all $(A, B) \in \mathcal{Q}(N)$, then $F_{\mu}$ is the Šipoš integral of the fuzzy measure $\nu$ given by $\nu(C)=\mu(C, \emptyset)$.

Proof : It is easy to check that if $\mu$ satisfies $\mu(A, B)=-\mu(B, A)$ then the Šipoš integral with respect to the capacity $\nu$ defined by $\nu(C)=\mu(C, \emptyset)$ satisfies the axioms (LBC), (MBC), (PWBC), (SPLBC $\left.{ }^{+}\right)$and $(\mathbf{S y m})$. Hence, by Theorem $3, F_{\mu}(w)=\breve{S}_{\nu}(w)$.

Consider now the asymmetric case.

Theorem 6 If $\mu$ satisfies $\mu(A, B)=\mu(N \backslash B, N \backslash A)$ for all $(A, B) \in \mathcal{Q}(N)$, then $F_{\mu}$ is the Choquet integral of the fuzzy measure $\nu$ given by $\nu(C)=$ $\mu(C, \emptyset)$.

Proof : It is easy to check that if $\mu$ satisfies $\mu(A, B)=\mu(N \backslash B, N \backslash A)$, then the Choquet integral with respect to the capacity $\nu$ defined by $\nu(C)=\mu(C, \emptyset)$ satisfies the axioms (LBC), (MBC), (PWBC), $\left(\mathbf{S P L B C}^{+}\right)$and $(\mathbf{S y m})$. Hence, by Theorem $3, F_{\mu}(w)=C_{\nu}(w)$.

\section{Discussion on the practicality of the method}

We have seen that the use of a bi-capacity enables to model more complex interaction behaviors between attractive and repulsive values than usual capacities. Some practical issues concerning the construction of $u_{i}$ and $\mu$ are considered in this section. 


\subsection{Construction of the reference levels}

We have assumed so far the existence of three reference levels $-\mathbf{1}_{i}, \mathbf{0}_{i}$ and $\mathbf{1}_{i}$ on each attribute $X_{i}$. Since their role is essential in the proposed method, it is useful to indicate how they can be determined.

The motivation of this paper which follows the one described in $[14,23]$ is to ask to the DM the least possible information to construct the full model. Starting right away with the most complicated model, namely a bi-capacity, without any knowledge regarding a non-symmetric behavior and thus that the usual Choquet model is not sufficient may not be the best strategy. We advise to begin with the unipolar assumption [23] (see Section 2.3). Two saturation levels are constructed on a unipolar scale. The $\mathbf{0}$ level corresponds to the total absence of some property, as the 0 level in fuzzy sets. Its determination can be derived as for fuzzy sets. The $\mathbf{1}$ reference level has the meaning of a satisfactory level. The existence of such a level has been the main argument of H. Simon in his theory of satisficing bounded rationality [34], and a fundamental assumption in the Macbeth methodology. These levels have been determined in a number of applications [2,3,4]. A methodology for their determination is proposed in [4]. Once the $\mathbf{0}_{i}$ and $\mathbf{1}_{i}$ levels are determined on the unipolar scales, one then gives some examples of comparisons between alternatives, exactly as in Section 3. If the Choquet integral fails to model those examples, one can think of more versatile aggregation functions. There may be really a lot of reasons for which a Choquet integral cannot represent a set of comparisons between acts. We are interested in this paper in the cases in which the underlying reason is non-symmetric attitude of the DM toward attractive and repulsive values. The example given in Section 3 is typical. The non-symmetric behavior is due to the presence of the neutral level. It has some consequences on the preference relation. The neutral elements $\mathbf{0}_{i}$ bear the very meaning of ratio scales. Hence, if non-symmetric phenomena occur in the expertise given by the DM, the neutral level does exist for the DM. So, he shall be able to provide this level.

Level $-\mathbf{1}_{i}$ is symmetric to level $\mathbf{1}_{i}$ and can be obtained in the same way. It may happen that there is no repulsive element on some attributes. In this case, it may be more complex to define the negative reference levels $-\mathbf{1}$ on these attributes. They can, however, be defined through the introduction of artificial elements added to the attributes, as done in the Macbeth approach [2]. This may be impossible for a DM, for instance if he cannot imagine an ill-satisfied value on a criterion. Let us denote by $I$ the set of criteria for which the $-\mathbf{1}_{i}$ elements cannot be defined. Let us investigate the practicality of our approach when $I \neq \emptyset$. Since $-\mathbf{1}_{i}$ is not defined for $i \in I$, bi-capacities 
are not defined on couples $(A, B)$ with $B \cap I \neq \emptyset$. Setting

$$
\mathcal{Q}_{I}(N)=\{(A, B) \in \mathcal{Q}(N), B \cap I=\emptyset\}
$$

we define $\mathcal{C}_{I}^{2}(N)$ as the restriction of bi-capacities to $\mathcal{Q}_{I}(N)$. Since attributes of $I$ have only attractive elements, the profile of acts of $X$ belong to

$$
\mathcal{R}_{I}=\left\{w=\left(w_{1}, \ldots, w_{n}\right) \in \mathbb{R}^{n}, \forall i \in I, w_{i} \geq 0\right\}
$$

It is easy to see that for $w \in \mathcal{R}_{I}$ and $\mu \in \mathcal{C}^{2}(N), B C_{\mu}(w)$ uses only terms of $\mu$ in $\mathcal{Q}_{I}(N)$. Hence, the Choquet integral can be defined in $\mathcal{R}_{I}$ from a "measure" in $\mathcal{C}_{I}^{2}(N)$. Our approach can be thoroughly extended to this case.

\subsection{Determination of the utility functions}

Let us investigate here how to construct in practice the utility function $u_{i}$. We follow the Macbeth methodology [1]. The goal of this section is not to give very precise details concerning the Macbeth approach. We refer to references [1] for a more detailed explanation of the Macbeth approach.

For the sake of simplicity, assume that attribute $X_{i}$ has a finite set of values : $X_{i}^{-}=\left\{a_{i}^{-q_{i}}, \ldots, a_{i}^{0}\right\}, X_{i}^{+}=\left\{a_{i}^{0}, \ldots, a_{i}^{p_{i}}\right\}$ with $a_{i}^{0}=\mathbf{0}_{i}$. We aim at determining $u_{i}^{-}\left(a_{i}^{j}\right)$ for all $j \in\left\{-q_{i}, \ldots, 0\right\}$ and $u_{i}^{+}\left(a_{i}^{j}\right)$ for all $j \in\left\{0, \ldots, p_{i}\right\}$.

From a theoretical standpoint, the construction of $u_{i}^{+}$(respectively $u_{i}^{-}$) from (Intra $\left.\mathbf{a}_{a}\right)$, (Intra $\left.{ }_{b}\right)$ and (Intra $\left.\mathbf{a}_{c}\right)$ is straightforward if the data is consistent. Indeed, applying (Intra ${ }_{b}$ ) with $x_{i}=a_{i}^{j}, y_{i}=z_{i}=\mathbf{0}_{i}$ and $w_{i}=\mathbf{1}_{i}$ (resp. $\left.w_{i}=-\mathbf{1}_{i}\right)$, together with $\left(\right.$ Intra $\left._{c}\right)$ yields

$$
u_{i}^{+}\left(a_{i}^{j}\right)=k^{+}\left(a_{j}^{i}, \mathbf{0}_{i}, \mathbf{1}_{i}, \mathbf{0}_{i}\right)\left(\operatorname{resp} . u_{i}^{-}\left(a_{i}^{j}\right)=k^{-}\left(a_{j}^{i}, \mathbf{0}_{i},-\mathbf{1}_{i}, \mathbf{0}_{i}\right)\right)
$$

where $k^{+}\left(\right.$resp. $\left.k^{-}\right)$is given by $\left(\operatorname{Intra}_{b}\right)$. Proceeding in this way ensures uniqueness of $u_{i}^{+}$and $u_{i}^{-}$.

Of course, it is not reasonable to ask a DM to give directly the value of $k^{\epsilon}$ as a real number. The idea of the Macbeth methodology is to ask an information of an ordinal nature to the DM, namely on the following ordinal scale $\{$ very small, small, mean, large, very large, extreme $\}=: \mathcal{E}$. In order to construct interval scales, the DM is asked to assess (giving a value in the scale $\mathcal{E})$ the difference of attractiveness $u_{i}^{+}\left(a_{i}^{j}\right)-u_{i}^{+}\left(a_{i}^{k}\right)$ (resp. $u_{i}^{-}\left(a_{i}^{j}\right)-u_{i}^{-}\left(a_{i}^{k}\right)$ ) between two values $a_{i}^{j}$ and $a_{i}^{k}$ in $X_{i}^{+}$(resp. $X_{i}^{-}$), for any $j \neq k$ such that $\left(\mathbf{0}_{-i}, a_{i}^{j}\right) \succ\left(\mathbf{0}_{-i}, a_{i}^{k}\right)$. The information asked in practice is thus quite similar to $\left(\operatorname{Intra}_{b}\right)$. The advantage of asking $u_{i}^{+}\left(a_{i}^{j}\right)-u_{i}^{+}\left(a_{i}^{k}\right)$ or $u_{i}^{-}\left(a_{i}^{j}\right)-u_{i}^{-}\left(a_{i}^{k}\right)$ is that it is easier for a human being to give some relative information regarding 
a difference (for instance $u_{i}^{+}\left(a_{i}^{j}\right)-u_{i}^{+}\left(a_{i}^{k}\right)$ or $\left.u_{i}^{-}\left(a_{i}^{j}\right)-u_{i}^{-}\left(a_{i}^{k}\right)\right)$ than to give some absolute information (for instance $u_{i}^{+}\left(a_{i}^{j}\right)$ or $u_{i}^{-}\left(a_{i}^{j}\right)$ ).

There is no unique utility functions $u_{i}^{+}$(resp. $u_{i}^{-}$) corresponding to the data composed of the values $u_{i}^{+}\left(a_{i}^{j}\right)-u_{i}^{+}\left(a_{i}^{k}\right)$ (resp. $u_{i}^{-}\left(a_{i}^{j}\right)-u_{i}^{-}\left(a_{i}^{k}\right)$ ), for $j \neq k$, given on the scale $\mathcal{E}$. All possible solutions have to be consistent with the given information. In practice, one utility function is chosen among all possible ones [1].

The drawback of asking redundant information is that some inconsistencies may be introduced by the DM. For instance, for $a_{i}^{j_{1}}, a_{i}^{j_{2}}$ and $a_{i}^{j_{3}}$ such that $\left(\mathbf{0}_{-i}, a_{i}^{j_{1}}\right) \succ\left(\mathbf{0}_{-i}, a_{i}^{j_{2}}\right) \succ\left(\mathbf{0}_{-i}, a_{i}^{j_{3}}\right)$, here is an example of inconsistency : the difference of satisfaction degree between $a_{i}^{j_{1}}$ and $a_{i}^{j_{2}}$ is judged very small by the DM, the one between $a_{i}^{j_{2}}$ and $a_{i}^{j_{3}}$ is also judged very small, and the one between $a_{i}^{j_{1}}$ and $a_{i}^{j_{3}}$ is judged extreme. C.A. Bana e Costa, and J.C. Vansnick showed that inconsistencies are related to cyclones in the preference relation structure [1]. This property enables to detect and explain all possible inconsistencies [1].

\subsection{Determination of the bi-capacity}

We could imagine proceeding as for $u_{i}$ to obtain $\mu$. The DM would be asked about the difference of satisfaction between the alternatives $\left(\mathbf{1}_{A},-\mathbf{1}_{B}, \mathbf{0}_{-A \cup B}\right)$ and $\left(\mathbf{1}_{C},-\mathbf{1}_{D}, \mathbf{0}_{-C \cup D}\right)$ (for $(A, B) \neq(C, D)$ ). Actually, even if this way is possible, it is not generally used because of the following two reasons. The first one is that it may not be natural for a DM to give his preferences on the prototypical acts $\left(\mathbf{1}_{A},-\mathbf{1}_{B}, \mathbf{0}_{-A \cup B}\right)$. The second one is that it enforces the DM to construct a ratio scale over $3^{n}$ alternatives. This requires roughly $\frac{\left(3^{n}\right)^{2}}{2}=\frac{9^{n}}{2}$ questions to asked to the DM. This is far too much in practice.

Conditions $\left(\operatorname{Inter}_{a}\right),\left(\mathbf{I n t e r}_{b}\right)$ and $\left(\mathbf{I n t e r}_{c}\right)$ were introduced to show the practicality of the use of the bi-capacities to model the preference relation $\succ$ on the Cartesian product of the attributes.

In practice, $\left(\mathbf{I n t e r}_{a}\right),\left(\mathbf{I n t e r}_{b}\right)$ and $\left(\mathbf{I n t e r}_{c}\right)$ shall be replaced by any classical method designed to deduce the bi-capacities from information on acts given in $\mathbb{R}^{n}$. One could think of natural extensions of methods determining capacities by optimization methods (linear programming $[26,27,13]$, quadratic programming [12], and heuristic-based methods [11]).

However, we are faced here with another difficulty. As mentioned previously, a bi-capacity contains $3^{n}$ unknowns which makes its determination quite delicate. As an example, with 5 criteria, a capacity has $2^{5}=32$ coefficients whereas a bi-capacity holds $3^{5}=243$ coefficients. Ten well-chosen 
learning examples are generally enough to determine a capacity with 5 criteria. It is not possible to say in advance the precise number of learning examples necessary to determine a bi-capacity. However, one can estimate that at least 80 learning examples are necessary for a bi-capacity related to 5 criteria. This is obviously beyond what a human being could stand.

The way out to this problem is to reduce the complexity of the model. The first idea is to restrict to sub-classes of bi-capacities, such as the $k$-additive bi-capacities [17]. For instance, there are $2 n^{2}=50$ unknowns for a 2 -additive bi-capacity with 5 criteria. Other approaches are also possible. One can for instance add new coefficients to a usual bi-capacities only when necessary and only in a limited context so that we do not go directly from $2^{n}$ to $3^{n}$ when usual capacities are not sufficient [24].

\section{Acknowledgment}

The authors wish to thank an anonymous referee for his careful reading and valuable suggestions, which helped to greatly improve the paper.

\section{References}

[1] C.A. Bana e Costa, J.C. Vansnick, A theoretical framework for Measuring Attractiveness by a Categorical Based Evaluation TecHnique (Macbeth), in: Proceedings of the XIth International Conference on MultiCriteria Decision Making, Portugal, 1994, pp.15-24.

[2] C.A. Bana e Costa, J.C. Vansnick, Applications of the MACBETH approach in the framework of an additive aggregation model, Journal of Multi-Criteria Decision Analysis 6 (1997) 107-114.

[3] C.A. Bana e Costa, F. Nunes da Silva, J.C. Vansnick, Conflict dissolution in the public sector: A case-study, European Journal of Operational Research 130 (2001) 388-401.

[4] C.A Bana e Costa, E.C. Correa, J. M. De Corte, J.C. Vansnick, Facilitating bid evaluation in public call for tenders: A socio-technical approach, Omega 30 (2002) 227-242.

[5] J.M. Bilbao, J.R. Fernandez, A. Jiménez Losada, E. Lebrón, Bicooperative games, in: Cooperative Game on Combinatorial structures. J.M. Bilbao (Eds.), Kluwer Academic Publisher, 2000. 
[6] H. Bleichrodt, John Miyamoto, A characterization of quality-adjusted life-years under Cumulative Prospect Theory, Mathematics of Operations Research 28(1) (2003) 181-193.

[7] G. Choquet, Theory of capacities, Annales de l'Institut Fourier 5 (1953) 131-295.

[8] D. Denneberg, Non-Additive Measure and Integral, Kluwer Academic Publisher, 1994.

[9] D. Felsenthal, M. Machover, Ternary voting games, International Journal of Game Theory 26 (1997) 335-351.

[10] J.C. Fodor, M. Roubens, Fuzzy preferences modeling and multicriteria decision aid, Kluwer Academic Publisher, 1994.

[11] M. Grabisch, A new algorithm for identifying fuzzy measures and its application to pattern recognition, in: International Joint Conference of the 4th IEEE International Conference on Fuzzy Systems and the 2nd International Fuzzy Engineering Symposium, Yokohama, Japan, March 1995, pp. 145-150.

[12] M. Grabisch, The application of fuzzy integrals in multicriteria decision making, European Journal of Operational Research 89 (1996) 445-456.

[13] M. Grabisch, T. Murofushi and M. Sugeno, Fuzzy measures and integrals, Physica-Verlag, Heidelberg, New York, 2000.

[14] M. Grabisch, Ch. Labreuche, J.C. Vansnick, On the Extension of Pseudo-Boolean Functions for the Aggregation of Interacting Criteria, European Journal of Operational Research 148 (2003) 28-47.

[15] M. Grabisch, Ch. Labreuche, The Symmetric and Asymmetric Choquet integrals in finite spaces for decision making, Statistical Letters 43 (2002) $37-52$.

[16] M. Grabisch, Ch. Labreuche, Bi-Capacities for decision making on bipolar scales, in: EUROFUSE Workshop on Information Systems, Varenna, Italy, September 2002, pp. 185-190.

[17] M. Grabisch, Ch. Labreuche, Bi-Capacities, in: Joint International Conference on Soft Computing and Intelligent Systems and 3d International Symposium on Advanced Intelligent Systems, Tsukuba, Japan, October 2002. 
[18] M. Grabisch, Ch. Labreuche, Bi-Capacities. Part II: The Choquet integral, Fuzzy Sets and Systems, to appear.

[19] S. Greco, B. Matarazzo, R. Slowinski, Bipolar Sugeno and Choquet integrals. in: EUROFUSE Workshop on Information Systems, Varenna, Italy, September 2002, pp. 117-144.

[20] R.L. Keeney, H. Raiffa, Decision with Multiple Objectives, Wiley, New York, 1976.

[21] E.P. Klement, R. Mesiar, E. Pap, Triangular Norms, Kluwer Academic Publishers, Dordrecht, 2000.

[22] D.H. Krantz, R.D. Luce, P. Suppes, A. Tversky, Foundations of measurement, vol 1: Additive and Polynomial Representations, Academic Press, 1971.

[23] Ch. Labreuche, M. Grabisch. The Choquet integral for the aggregation of interval scales in multicriteria decision making, Fuzzy Sets and Systems 137 (2003) 11-26.

[24] Ch. Labreuche, M. Grabisch, Partially unipolar bi-capacities in MCDM, in: SCIS-ISIS 2004, 2nd International Conference on Soft Computing and Intelligent Systems and 5th International Symposium on Advanced Intelligent Systems, Yokohama, Japan, September 2004.

[25] J.L. Marichal, An axiomatic approach of the discrete Choquet integral as a tool to aggregate interacting criteria, IEEE Transactions on Fuzzy Systems 8(6) (2000) 800-807.

[26] J.L. Marichal, M. Roubens, Dependence between criteria and multiple criteria decision aid, in: 2nd International Workshop on Preferences and Decisions, Trento, Italy, 1998, pp. 69-75.

[27] J.L. Marichal, M. Roubens, On a sorting procedure in the presence of qualitative points of view, in: J. Chojean and J. Leski (Eds.), Fuzzy Sets and Their Applications, Silesian University Press, Gliwice, Poland, 2001, pp. 217-230.

[28] C.E. Osgood, G.J. Suci, P.H. Tannenbaum, The measurement of meaning, University of Illinois Press, Urbana, IL, 1957.

[29] G. Owen, Game Theory, Academic Press, San Diego, third edition, 1995. 
[30] J.C. Pomerol, S. Barba-Romero, Multicriterion decision in management: principles and practice, Kluwer Academic Publishers, 2000.

[31] D. Schmeidler, Integral representation without additivity, Proceedings of the American Mathematical Society 97 (1986) 255-261.

[32] D. Schmeidler, Subjective probability and expected utility without additivity, Econometrica 57(3) (1989) 571-587.

[33] L.S. Shapley, Simple games: an outline of the descriptive theory, Behavioral Science 7 (1962) 59-66.

[34] H. Simon, Rational choice and the structure of the environment, Psychological Review 63(2) (1956) 129-138.

[35] P. Slovic, M. Finucane, E. Peters, D.G. MacGregor, The affect heuristic. in: T. Gilovitch, D. Griffin, D. Kahneman (Eds.), Heuristics and biases: the psychology of intuitive judgment, Cambridge University Press, 2002, pp. 397-420.

[36] J. Šipoš, Integral with respect to a pre-measure, Mathematical Slovaca 29 (1979) 141-155.

[37] A. Tversky, D. Kahneman, Advances in Prospect theory: Cumulative Representation of Uncertainty, Journal of Risk and Uncertainty 5 (1992), pp. 297-323.

[38] P. Wakker, A. Tversky, An axiomatization of Cumulative Prospect Theory, Journal of Risk and Uncertainty 7 (1993) 147-176.

[39] H. Zank, Cumulative Prospect Theory for parametric and multiattribute utilities, Mathematics of Operations Research 26(1) (2001) 67-81. 